\title{
Dissipatividade em Sistemas Dinâmicos Fuzzy Discretos
}

\section{Suzana Abreu de Oliveira Souza}

TESE APRESENTADA AO INSTITUTO DE MATEMÁTICA E ESTATÍSTICA

DA UNIVERSIDADE DE SÃO PAULO PARA OBTENÇÃO DO GRAU DE DOUTOR EM MATEMÁTICA APLICADA

Área de Concentração: Matemática Aplicada Orientador: Prof. Dr. Pedro Aladar Tonelli 


\section{Dissipatividade em Sistemas Dinâmicos Fuzzy Discretos}

Estc excmplar corresponde à redação da tese devidamente corrigida e defendida por Suzana Abreu de Oliveira Souza.

São Paulo, abril de 2001.

Banca examinadora

- Prof Dr. Pedro Aladar Tonelli (Orientador) IME USP

- Prof Dr. Luciano Barbanti IME USP

- Prof Dr. Fernando Antonio Campos Gomide FEEC UNICAMP

- Prof Dr. Laécio Carvalho de Barros IMECC UNICAMP

- Prof Dr. Rodney Carlos Bassanezi IMECC UNICAMP 
À minha familia

$e$ aos meus amigos 


\section{Agradecimentos}

Primeiramente a Deus, sem o qual nada do que fazemos tem sentido. Aos meus amigos Laécio e Pedro, pela paciência que tiveram comigo e porque me ensinaram a caminhar. Ao meu marido e ao meu filho pela compreensão nas ausências. À minha mãe que me deu condições para chegar até aqui e à Neli Regina que muitas vezes teve que aguentar os meus seminários. 


\section{Resumo}

Neste trabalho estudamos a dissipatividade e a suavidade assintótica de extensões de Zadeh de uma função contínua em $\mathbb{R}^{n}$ que dá origem a um Sistema Dinâmico Discreto. Apresentamos uma condição necessária para que esta extensão seja assintoticamente suave no espaço dos conjuntos fuzzy. Esta propriedade é relevante para o estudo de atratores em Sistemas Dinâmicos. Palavras-chave: Sistemas Dinâmicos Fuzzy, Dissipatividade, Conjuntos Fuzzy.

\section{Abstract}

In this work we study the dissipativity and asymptotic smoothness of Zadeh's extension of a continuous map on $\mathbb{R}^{n}$ that generates a Discrete Dynamical System. We present a necessary condition to this extension be asymptotically smooth in the space of fuzzy sets. This property is relevant to study atractors in Dynamical Systems.

Key-words: Fuzzy Dynamical Systems, Dissipativity, Fuzzy Sets. 


\section{Conteúdo}

1 Introdução

2 Espaço de Conjuntos Fuzzy 7

2.1 Introdução . . . . . . . . . . . . . . . . 7

2.2 Conjuntos Fuzzy . . . . . . . . . . . . . . . 8

2.3 Sobre T-Normas e T-Conormas . . . . . . . . . . . 14

2.3 .1 T-Normas . . . . . . . . . . . . . . 14

2.3 .2 T-Conormas . . . . . . . . . . . . . 16

2.3.3 Generalização de Complementar de um Conjunto Fuzzy 17

2.3.4 Combinação de Operações . . . . . . . . . . . . . . 19

2.4 Níveis de um Conjunto Fuzzy . . . . . . . . . . . . . . 20

2.5 Métricas no Espaço dos Conjuntos Fuzzy . . . . . . . . . . . 24

2.6 Convergências em $\mathcal{F}\left(\mathbb{R}^{n}\right) \ldots \ldots \ldots \ldots . \ldots \ldots$

3 A Extensão de Zadeh e Propriedades 32

3.1 O Princípio da Extensão . . . . . . . . . . . . . . . . 32

3.2 Propriedades da Extensão . . . . . . . . . . . . . . . 34

3.3 Extensão usando T-Conorma e T-Norma . . . . . . . . . . . 39

4 Sistemas Dinâmicos Discretos e Dissipatividade 41

4.1 Sistemas Dinâmicos Discretos . . . . . . . . . . . . . 41

4.2 Aplicações Assintoticamente Suaves . . . . . . . . . . 45

4.3 Dissipatividade . . . . . . . . . . . . . . 47

5 Dissipatividade em Sistemas Dinâmicos Fuzzy e Aplicações 51

5.1 Introdução . . . . . . . . . . . . . . . . . . 51

5.2 Sobre Conjuntos Compactos em $\mathcal{F}\left(\mathbb{R}^{n}\right) \ldots \ldots \ldots \ldots 1$

5.3 Alguns Resultados sobre Sistemas Dinâmicos Fuzzy . . . . . 55 
5.4 O Problema da Suavidade Assintótica em $\mathcal{F}\left(\mathbb{R}^{n}\right) \ldots \ldots$. . . 57

5.5 Um Exemplo em $\mathcal{F}^{*}\left(\mathbb{R}^{n}\right) \ldots \ldots \ldots \ldots \ldots . \ldots \ldots$

5.6 Outros Exemplos . . . . . . . . . . . . . . . . 64

5.7 Dissipatividade em $\mathcal{F}\left(\mathbb{R}^{n}\right) \ldots \ldots \ldots 6$. . . . . . . . . . . . . 65

5.8 Projetos .......................... 68 


\section{Capítulo 1}

\section{Introdução}

"Quanto mais de perto encaramos um problema na vida real, mais nebulosa se torna a sua soluçâo".

Lofti Zadeh

Nebuloso, vago, incerto são conceitos que incomodam a humanidade desde os primórdios. Problemas como: "Um homem tem a cabeça repleta de cabelos e começa-se a retirar fio por fio, e a cada fio retirado pergunta-se se ele está calvo. Quando classificá-lo como calvo?" Neste processo ele ficará calvo e até careca. Será que podemos dizer qual o fio que determina a calvice ou não do homem? Paradoxos como este existem desde a Grécia antiga. Este, particularmente, foi apresentado por Bertrand Russell no início do século vinte.

Foi também nesta época, em 1937, que um filósofo chamado Max Black publicou o artigo "Vagueness: An Exercise in Logical Analysis" [7]. Seu artigo definia uma curva que agora identificamos com a função pertinência de um conjunto fuzzy. Max Black usou o termo "vago" porque os filósofos da época, como Bertrand Russell, o usavam para chamar o que hoje nós chamamos de "fuzzy". Na época, ninguém deu atenção ao artigo de Black, sendo fadado ao esquecimento. Alguns anos depois, em 1965, Lofti A. Zadeh, natural de Baku no Irã, como também Max Black, lançou seu primeiro artigo chamado "Fuzzy Sets" [38], mas ao contrário de Black, Zadeh era um proeminente professor da U. C. Berkeley e sua influência ajudou bastante na divulgação de suas idéias, tanto que hoje ele é considerado o precursor da Teoria Fuzzy.

A partir de então, teorias e aplicações têm surgido e algumas das principais áreas que encontramos a teoria fuzzy são: Sistemas de Controles Fuzzy, 
Tomada de Decisão, Reconhecimento de Padrões e Processamento de Imagens, Medicina e Biologia. Na medicina, por exemplo, os principais focos têm sido a modelagem dos processos de diagnósticos de doenças [35] e epidemologia [24]. Na Biologia, modela-se a dinâmica de população. Em Barros [2], são consideradas basicamente duas situações: A primeira quando acredita-se que o meio onde vivem os indivíduos interfere fortemente na dinâmica da população e outra trata-se de classificar presa e predador, onde estes conceitos são apresentados baseando-se na teoria fuzzy.

O sucesso das aplicações motivou o desenvolvimento da teoria fuzzy e, um dos pontos cruciais deste aprofundamento teórico foi o estudo das Equações Diferenciais Fuzzy, que generalizam o conceito de Equações Diferenciais Ordinárias, no sentido que os campos destas equações são extensões de multifunções e estas estendem as funções clássicas. Kaleva [16], Seikkala [36], Ralescu [22], dentre outros, desenvolveram ferramentas analíticas para o estudo de um problema de Cauchy num espaço de conjuntos fuzzy. As aplicações deste conceito contudo, esbarram na falta das boas propriedades dos fluxos obtidos das Equações Diferencias Fuzzy, como por exemplo, a estabilidade da solução, já que a solução de uma Equação Diferencial Fuzzy tem o diâmetro de seus níveis crescentes, até mesmo a solução da seguinte equação:

$$
\dot{u}=-\boldsymbol{u}
$$

como pode ser visto em Barros [3]. Um outro caminho tem sido estudar as Inclusões Diferenciais Fuzzy, onde procura-se $u(t)$ tal que:

$$
u^{\prime}(t) \in[G(t, u(t))]^{\alpha},
$$

onde $G$ é uma função com valores em conjuntos fuzzy, $[G(t, u(t))]^{\alpha}$ são subconjuntos compactos do $\mathbb{R}^{n}$ conhecidos como $\alpha$-níveis do conjunto fuzzy $G(t, u(t))$ e $u$ ' é a derivada de $u$ segundo Hukuhara [12].

No caso de Sistemas Dinâmicos, com relação à teoria determinística, a teoria fuzzy abre um campo maior de aplicações, já que esta pode modelar fenômenos que apresentam algum tipo de incerteza. No entanto, para fenômenos onde as incertezas são desprezadas, se considerarmos os dois Sistemas Dinâmicos, a solução determinística também é solução do Sistema Dinâmico Fuzzy e é chamada de preferida, conforme Barros [3].

Neste trabalho, consideramos a teoria de Sistemas Dinâmicos Fuzzy Discretos, obtidos a partir de um Sistema Dinâmico Discreto Determinístico. 
Dois aspectos são relevantes aqui: A topologia do espaço métrico de conjuntos fuzzy, $\mathcal{F}\left(\mathbb{R}^{n}\right)$, e a teoria de Sistemas Dinâmicos em espaços de dimensão infinita. A topologia porque procuramos compactos em $\mathcal{F}\left(\mathbb{R}^{n}\right)$, para estudar a dissipatividade de sistemas neste espaço, que é de dimensão infinita.

Lembrando que um Sistema Dinâmico Discreto é um sistema iterativo [14]

$$
x_{n+1}=f\left(x_{n}\right) \text {, }
$$

onde $f: X \rightarrow X$ é uma função dada e $x_{o} \in X$, estudamos Sistemas Dinâmicos em que os pontos são substituídos por subconjuntos fuzzy $u \in$ $\mathcal{F}(X)$ e a função $f$ é estendida para $\hat{f}: \mathcal{F}(X) \rightarrow \mathcal{F}(X)$. Assim, o sistema dinâmico fuzzy discreto associado a (1.3) é dado por:

$$
u_{n+1}=\hat{f}\left(u_{n}\right) \text {. }
$$

No nosso caso, partimos de um sistema dinâmico onde a função dada $f$ está definida em $\mathbb{R}^{n}$, ou seja, $f: \mathbb{R}^{n} \rightarrow \mathbb{R}^{n}$, e é contínua. O nosso objetivo é investigar quando a sua extensão, $\hat{f}: \mathcal{F}\left(\mathbb{R}^{n}\right) \rightarrow \mathcal{F}\left(\mathbb{R}^{n}\right)$, é uma aplicação assintoticamente suave.

Estudar a suavidade assintótica ou dissipatividade da função que dá origem a um sistema dinâmico discreto é de suma importância para a investigação de atratores e o estudo de seu comportamento assintótico, por exemplo, se em seu domínio existe um conjunto $J$ compacto e invariante que atrai pontos localmente, então $J$ atrai compactos em uma vizinhança de $J$, ver [14], página 12 .

No caso, sabemos que $f: \mathbb{R}^{n} \rightarrow \mathbb{R}^{n}$ é assintoticamente suave, já que $f$ é contínua e no espaço $\mathbb{R}^{n}$, todo conjunto fechado e limitado é compacto. Para estudar a extensão $\hat{f}$, reduzimos o problema aos $\alpha$-níveis de um conjunto fuzzy, já que estes $\alpha$-níveis são conjuntos compactos do $\mathbb{R}^{n}$. Os $\alpha$-níveis de um conjunto fuzzy são os seguintes conjuntos:

$$
[u]^{\alpha}=\left\{x \in \mathbb{R}^{n} / u(x) \geq \alpha\right\}, \text { se } 0<\alpha \leq 1
$$

e

$$
[u]^{0}=\overline{\left\{x \in \mathbb{R}^{n} / u(x)>0\right\}} .
$$

Acreditando que esta extensão seria sempre assintoticamente suave, procuramos construir um compacto candidato a atrator em $\mathcal{F}\left(\mathbb{R}^{n}\right)$. Resultado este alcançado quando nos restringimos ao espaço dos conjuntos fuzzy cujos $\alpha$-níveis não têm pontos de máximos locais próprios com níveis 1 contando 
apenas com um elemento, $\mathcal{F}^{*}\left(\mathbb{R}^{n}\right)$, (pág. 61), ou seja, quando asseguramos que os $\omega$-limites de todos os conjuntos fechados e limitados do domínio da $\hat{f}$ são não-vazios, então temos o compacto que precisamos para mostrar que neste espaço a extensão é assintoticamente suave.

A partir do seguinte resultado encontrado em [31], que diz que

$$
J=\left\{u \in \mathcal{F}\left(\mathbb{R}^{n}\right) /[u]^{o} \subset K\right\}
$$

é compacto se, e somente se $K$ tem diâmetro zero, extraimos outras propriedades do conjunto que gostaríamos que fosse compacto, e um resultado interessante mostrado na pág. 60, diz: "Se J dado como acima for vazio, então $\hat{f}$ não é assintoticamente suave".

O trabalho está organizado da seguinte maneira: No capítulo 2 nos preocupamos em falar com detalhes sobre conjuntos fuzzy, suas propriedades, suas operações aritméticas e compará-los com a Teoria Clássica de Conjuntos . Fizemos algumas ilustrações para esclarecer melhor os exemplos. Apresentamos ainda neste capítulo generalizações para interseções e uniōes de conjuntos fuzzy através de T-normas e T- conormas, também apresentando exemplos.

No capítulo 3, apresentamos o Princípio da Extensão, outras extensões usando interseções e uniões através de T-normas e T-conormas, a Extensão de Zadeh e suas principais propriedades que são bastante estudadas em [1] [6], [28], [35].

No capítulo 4, estudamos a teoria clássica de Sistemas Dinâmicos Discretos apresentada por Jack Hale [14], onde a teoria será aplicada em Sistemas Dinâmicos Fuzzy no capítulo seguinte.

No capítulo 5 então, apresentamos alguns resultados sobre Sistemas Dinâmicos Discretos Fuzzy, aplicamos a teoria apresentada por Jack Hale para obter os resultados de dissipatividade em $\mathcal{F}\left(\mathbb{R}^{n}\right)$ e $\mathcal{F}^{*}\left(\mathbb{R}^{n}\right)$ e apresentamos aplicações através de exemplos. 


\section{Capítulo 2}

\section{Espaço de Conjuntos Fuzzy}

Neste capítulo vamos apresentar a definição de conjuntos fuzzy, com as métricas que vamos trabalhar bem como alguns tipos de convergências e suas equivalências.

\subsection{Introdução}

Quando queremos classificar uma figura geométrica plana podemos nos referir a alguns conjuntos, como: $O$ conjunto das circunferências, o dos triângulos, o dos retângulos, o dos quadrados e assim por diante. Olhando uma figura geométrica plana, através de suas características, podemos dizer com toda a certeza se ela pertence ao conjunto dos triângulos ou não. Mas nem todas as classificações seguem estes parâmetros. Por exemplo: Se quisermos classificar pessoas pela altura, ou seja, pessoas altas e pessoas baixas, estes conjuntos não estão tão bem definidos, como os das figuras geométricas planas citados acima. Dependendo do lugar geográfico em que estamos fazendo a classificação, estes parâmetros podem mudar, e muito. Digamos que $x$ é a altura em metros de um indivíduo e que o conjunto $A$ das pessoas baixas está definido da seguinte maneira:

$$
A=\{x ; x<1,60 m\}
$$

A pergunta é: Uma pessoa que tem $1,30 \mathrm{~m}$ é tão baixa quanto uma pessoa que mede $1,59 \mathrm{~m}$ ? No entender cotidiano, não. Daí a necessidade de criar um novo conceito de conjunto e pertinência neste conjunto. 
A noção de conjunto fuzzy (que quer dizer: vago, nebuloso), foi introduzida por Zadeh [38] em 1965 e generaliza o conceito de pertinência de um elemento ao conjunto "medindo" seu "grau de pertinência" através de uma aplicação que vai do conjunto cujos elementos devem ser classificados, no intervalo fechado $[0,1]$.

Vamos dar dois exemplos:

Seja $A$ um subconjunto de um conjunto clássico $X$ e considere a função característica:

$$
\chi_{A}(x)=\left\{\begin{array}{l}
1 \text { se } x \in A \\
0, \text { caso contrário. }
\end{array}\right.
$$

Então a função característica é uma função pertinência $\chi_{A}$, que assume valores 0 ou 1, indica quando um elemento $x \in X$ é também elemento de $A$, ou seja, $\chi_{A}(x)=1$ se $x \in A$ e $\chi_{A}(x)=0$ se $x \notin A$.

Agora considere $A=\{x ; x<1,60 m\}$ como acima. Neste caso, se tiver$\operatorname{mos} u: X \rightarrow[0,1]$, dada por

$$
u(x)=\left\{\begin{array}{l}
\frac{-10}{16} x+1 \text { se } 0 \leq x<1,60 \\
0 \text { se } x \geq 1,60
\end{array}\right.
$$

como a função que indica "o quanto $x$ pertence a $A$ ", podemos dizer que a pessoa de $1,30 \mathrm{~m}$ tem grau de pertinência $u(1,30)=\frac{3}{16}$ e a pessoa de 1,59 m tem grau de pertinência $u(1,59)=\frac{1}{160}$ ao conjunto fuzzy gerado por $A$. Desta forma, podemos dizer qual o grau de pertinência destas pessoas com relação ao conjunto $A$, ou seja, a primeira pessoa pertence mais do que a segunda pessoa.

Passamos então a formalização deste conceito.

\subsection{Conjuntos Fuzzy}

Seja $X$ um conjunto clássico e $u: X \rightarrow[0,1]$ uma aplicação de $X$ no intervalo real $[0,1]$, que chamaremos de função grau de pertinência ou apenas função pertinência. Um subconjunto fuzzy de $X$ é um conjunto de pares ordenados $\{(x, u(x)) ; x \in X\}$. Aqui iremos representar o conjunto fuzzy pela sua função pertinência $u$ e chamaremos subconjuntos fuzzy apenas de conjuntos fuzzy.

A seguir, vamos ilustrar o conceito de conjunto fuzzy com alguns exemplos. 
Exemplo 2.1 Seja $X=\mathbb{R}$ e $u: \mathbb{R} \rightarrow[0,1]$ dada por: $u(x)=\left\{\begin{array}{l}x^{2}, \text { se } x \in[0,1] \\ 0, \text { caso contrário }\end{array}\right.$

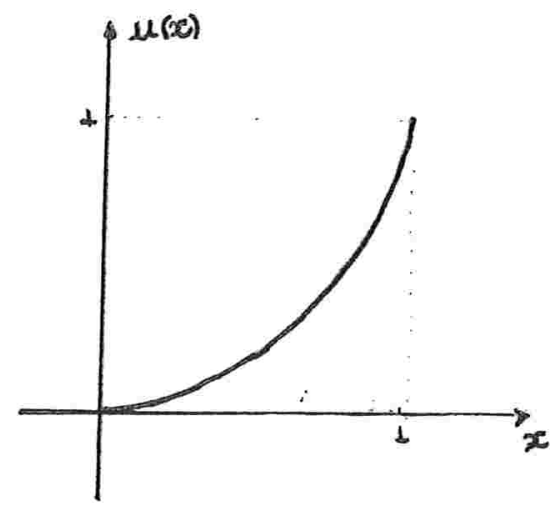

Podemos também ter uma seqüência de conjuntos fuzzy.

Exemplo 2.2 Seja $X=\mathbb{R}$, para $n \geq 1$, definamos:

$u_{n}(x)=\left\{\begin{array}{l}\frac{x}{n}+1-\frac{1}{n} \text { se } x \in[0,1] \\ 0, \text { caso contrário }\end{array}\right.$

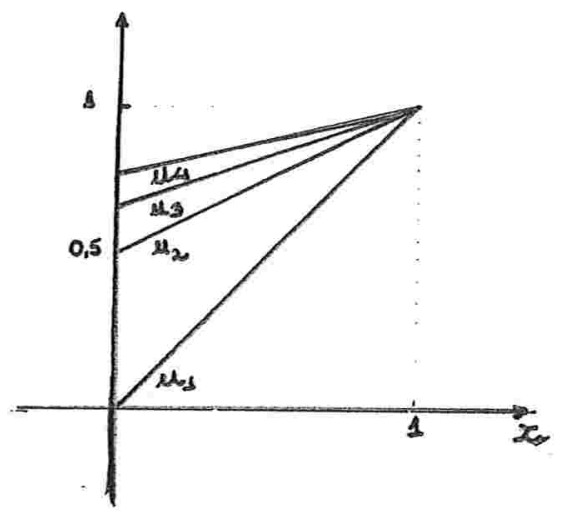


Vamos enumerar algumas definições envolvendo conjuntos fuzzy e que são, na verdade, extensões das definições correspondentes de conjuntos clássicos.

Seja $X$ um conjunto clássico e $A$ um subconjunto $X$.

Um subconjunto fuzzy $A$ é dito vazio se sua função pertinência é identicamente nula.

Dois subconjuntos fuzzy $A$ e $B$ são iguais se as funções pertinências são iguais, ou seja, se $u_{A}(x)=u_{B}(x)$, para todo $x \in X$.

O complementar $A^{\prime}$ de um subconjunto fuzzy $A$ é definido pela função pertinência $u_{A^{\prime}}(x)=1-u_{A}(x)$, vejamos o exemplo abaixo:

Exemplo 2.3 Seja $X=\mathbb{R}$ e $A$ o conjunto das pessoas jovens, onde:

$$
u_{A}(x)=\left\{\begin{array}{l}
1 \text { se } x<25 \\
\frac{40-x}{15} \text { se } 25 \leq x \leq 40 \\
0 \text { se } 40<x
\end{array}\right.
$$

Então a função pertinência de A'é dada por:

$$
u_{A^{\prime}}(x)=\left\{\begin{array}{l}
0 \text { se } x<25 \\
\frac{x-25}{15} \text { se } 25 \leq x \leq 40 \\
1 \text { se } 40<x
\end{array}\right.
$$

Ou seja, o complementar de pessoas jovens definido pelo conjunto acima, são pessoas não jovens (acima de 25 anos). Vejamos pelos gráficos abaixo:
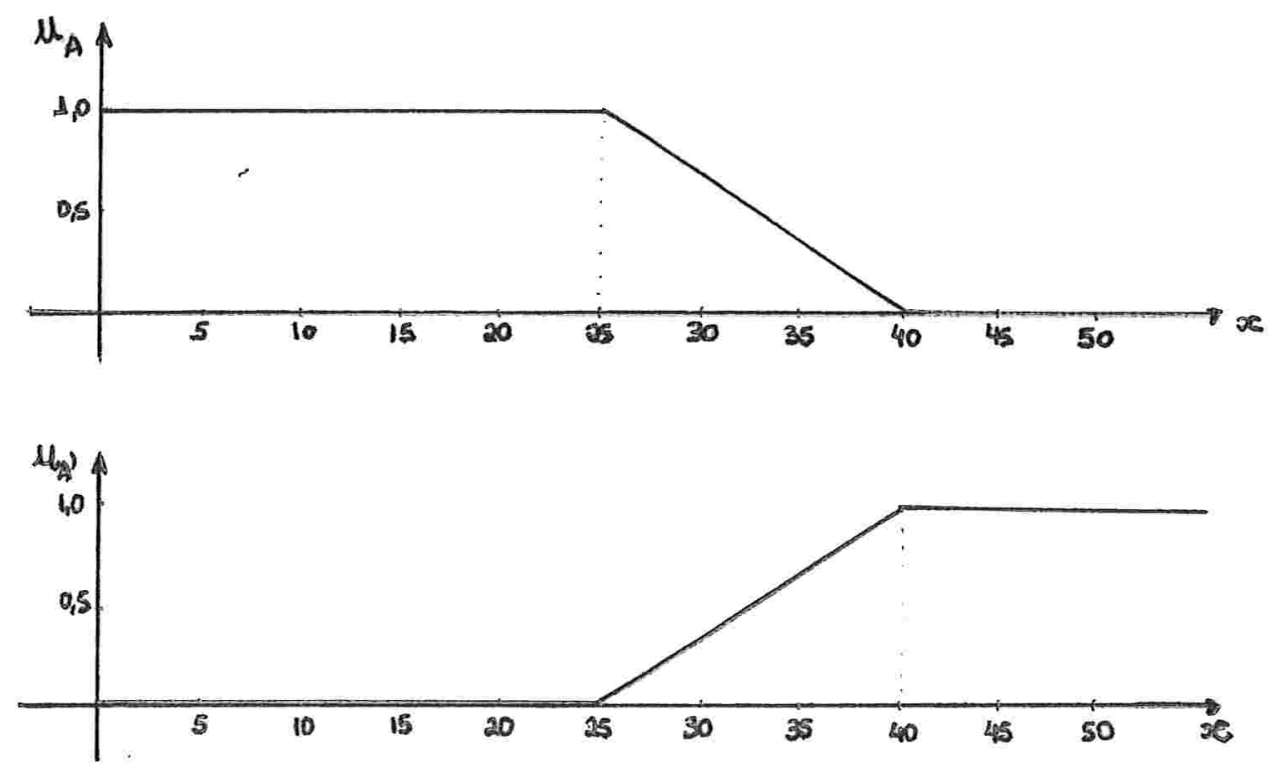
Como na teoria clássica de conjuntos, existe uma relação entre conjuntos muito importante na teoria de conjuntos fuzzy. Dizemos que $A$ está contido em $B$, ou seja, $A$ é subconjunto fuzzy de $B$, e representa-se por $A \subset B$, se as funções pertinências satisfazem à seguinte desigualdade:

$$
u_{A}(x) \leq u_{B}(x), \forall x \in X .
$$

Dados $A$ e $B$ conjuntos fuzzy, definimos o conjunto $C$ união de $A$ e $B$, ou seja, $C=A \cup B$, pela função pertinência dada por:

$$
u_{C}(x)=\max \left\{u_{A}(x), u_{B}(x)\right\}, \forall x \in X .
$$

Observação 2.1 Uma maneira mais intuitiva de definir o conjunto união seria dizer que a união entre $A$ e $B$ é o menor conjunto fuzzy contendo ambos $A$ e $B$. Mais precisamente, se $D$ é um conjunto fuzzy que contém $A$ e $B$, então $D$ contém a união.

Para mostrar que esta definição é equivalente à anterior, notemos que o conjunto definido anteriormente contém $A$ e $B$, já que

$$
u_{A}(x) \leq \max \left\{u_{A}(x), u_{B}(x)\right\}
$$

e

$$
u_{B}(x) \leq \max \left\{u_{A}(x), u_{B}(x)\right\}
$$

$\mathrm{E}$, se $D$ é um conjunto fuzzy que contém $A$ e $B$, então

$$
u_{A}(x) \leq u_{D}(x), \forall x \in X
$$

$\mathrm{e}$

$$
u_{B}(x) \leq u_{D}(x), \forall x \in X
$$

então

$$
\max \left\{u_{A}(x), u_{B}(x) \leq u_{D}(x)\right\}=u_{C}(x), \forall x \in X,
$$

o que implica que $C \subset D$.

A interseção de dois conjuntos fuzzy $A$ e $B$ representada por $C=A \cap B$ tem função pertinência dada por:

$$
u_{C}(x)=\min \left\{u_{A}(x), u_{B}(x)\right\}, \forall x \in X .
$$

Como no caso da união, é simples mostrar que o conjunto interseção definido como acima é o maior conjunto fuzzy contido em $A$ e $B$.

Com as definições acima, vemos que as mesmas propriedades de conjuntos clássicos se preservam. 
Teorema 2.1 Sejam $A, B$ conjuntos fuzzy de $X$. Então valem as seguintes propriedades:

1. $(A \cup B) \cup C=A \cup(B \cup C)$ - associativa da união

2. $(A \cap B) \cap C=A \cap(B \cap C)$ - associativa da interseçẫo

3. $C \cap(A \cup B)=(C \cap A) \cup(C \cap B)$ - distributiva

4. $C \cup(A \cap B)=(C \cup A) \cap(C \cup B)$ - distributiva

5. $(A \cup B)^{\prime}=A^{\prime} \cap B^{\prime}$ - lei de De Morgan

6. $(A \cap B)^{\prime}=A^{\prime} \cup B^{\prime}$ - lei de De Morgan

\section{Dem.:}

Apenas para ilustrar, vamos dar as idéias das demonstrações dos itens 4 e 5 .

Em 4), $C \cup(A \cap B)$, em termos de função pertinência é dado por:

$$
\max \left\{u_{C}(x), \min \left\{u_{A}(x), u_{B}(x)\right\}\right\}, \forall x \in X
$$

para mostrar que é igual a

$$
\min \left\{\max \left\{u_{A}(x), u_{C}(x)\right\}, \max \left\{u_{B}(x), u_{C}(x)\right\}\right\}, \forall x \in X
$$

devemos considerar os seis casos abaixo:

- $u_{A}(x) \geq u_{B}(x) \geq u_{C}(x)$

- $u_{A}(x) \geq u_{C}(x) \geq u_{B}(x)$

- $u_{B}(x) \geq u_{A}(x) \geq u_{C}(x)$

- $u_{B}(x) \geq u_{C}(x) \geq u_{A}(x)$

- $u_{C}(x) \geq u_{A}(x) \geq u_{B}(x)$

- $u_{C}(x) \geq u_{B}(x) \geq u_{A}(x)$

Então, se dado $x \in X$, tivermos $u_{A}(x) \geq u_{B}(x) \geq u_{C}(x)$ :

$\max \left\{u_{C}(x), \min \left\{u_{A}(x), u_{B}(x)\right\}\right\}=\max \left\{u_{C}(x), u_{B}(x)\right\}=u_{B}(x) \mathrm{e}$ $\min \left\{\max \left\{u_{A}(x), u_{C}(x)\right\}, \max \left\{u_{B}(x), u_{C}(x)\right\}\right\}=$

$\min \left\{u_{A}(x), u_{B}(x)\right\}=u_{B}(x)$.

Verificando-se os outros cinco casos, vemos que vale a igualdade.

Para 5), deve-se mostrar que:

$$
1-\max \left\{u_{A}(x), u_{B}(x)\right\}=\min \left\{1-u_{A}(x), 1-u_{B}(x)\right\}, \forall x \in X .
$$


Neste caso, temos apenas duas possibilidades:

- $u_{A}(x) \geq u_{B}(x)$

- $u_{B}(x) \geq u_{A}(x)$

Suponhamos que $u_{A}(x) \geq u_{B}(x)$ :

$1-\max \left\{u_{A}(x), u_{B}(x)\right\}=1-u_{A}(x) \mathrm{e}$ $\min \left\{1-u_{A}(x), 1-u_{B}(x)\right\}=1-u_{A}(x)$.

A demonstração se completa verificando-se a segunda possibilidade.

O exemplo abaixo ilustra graficamente a interseção e a união de conjuntos fuzzy.

Exemplo 2.4 Sejam

$$
u_{A}(x)=\left\{\begin{array}{l}
1 \text { se } x<25 \\
\frac{40-x}{15} \text { se } 25 \leq x \leq 40 \\
0 \text { se } 40<x
\end{array}\right.
$$

$e$

$$
u_{B}(x)=\left\{\begin{array}{l}
0 \text { se } x<25 \\
\frac{x-25}{15} \text { se } 25 \leq x \leq 40 \\
1 \text { se } 40<x
\end{array}\right.
$$
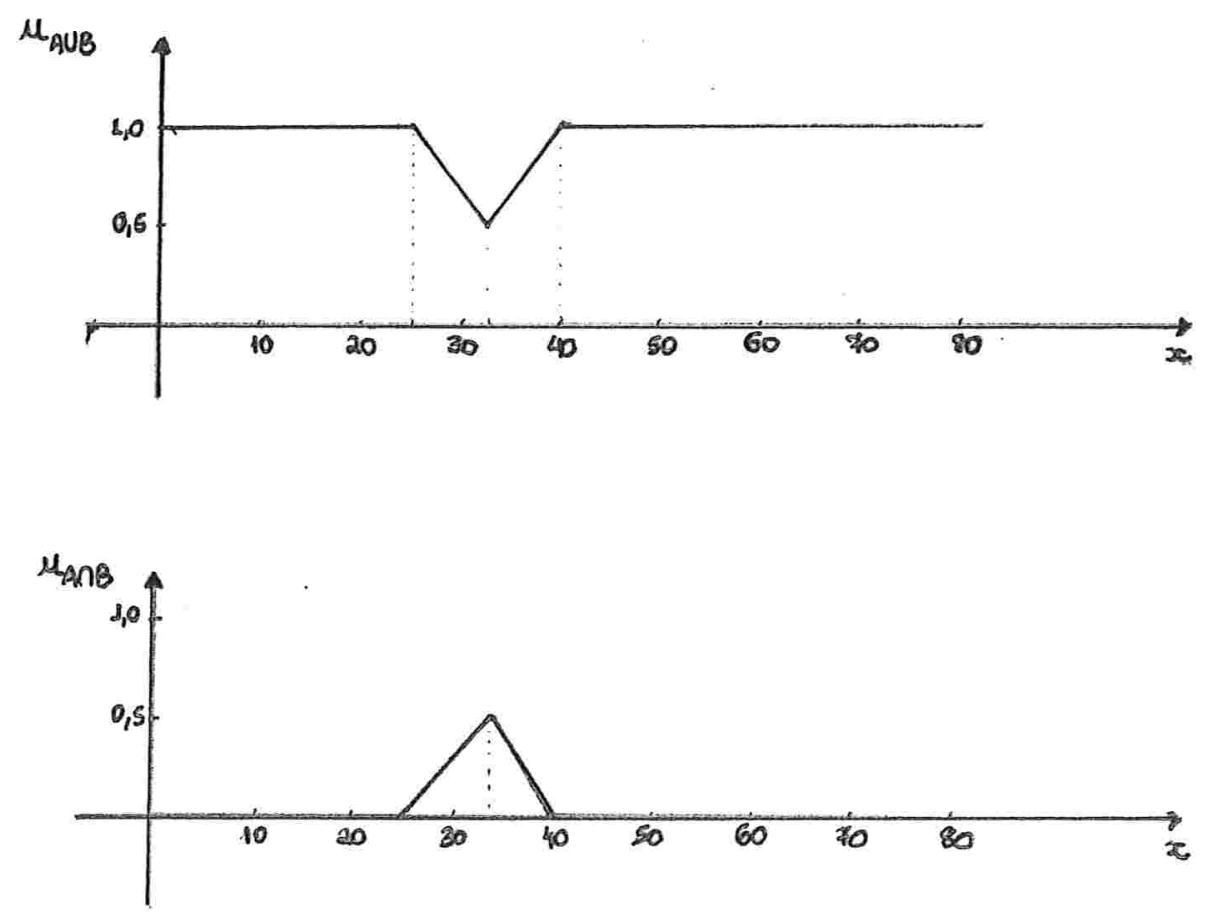


\subsection{Sobre T-Normas e T-Conormas}

\subsubsection{T-Normas}

Definição 2.1 Dizemos que uma aplicação $T:[0,1] \times[0,1] \rightarrow[0,1]$ é uma T-norma se:

1. $T(1, a)=a, \forall a \in[0,1]$

2. $T(a, b)=T(b, a), \forall a, b \in[0,1]$

3. Se $a \leq c$ e $b \leq d$ então $T(a, b) \leq T(c, d)$

4. $T(a, T(b, c))=T(T(a, b), c), \forall a, b, c \in[0,1]$

Exemplo 2.5 Seja $T:[0,1] \times[0,1] \rightarrow[0,1]$ tal que $T(a, b)=\min \{a, b\}$, $\forall a, b \in[0,1]$. Esta aplicação é uma T-norma.

De fato,

1. se $0 \leq a \leq 1$ então $\min \{1, a\}=a$;

2. $\min \{a, b\}=\min \{b, a\}$;

3. Se $d \leq c$ e $a \leq b$ então $\min \{a, d\} \leq \min \{c, b\}$, pois:

$$
\min \{d, a\} \leq \min \{c, a\} \leq \min \{c, b\}
$$

4. Devemos considerar os seis casos abaixo:

$a \leq b \leq c$
$-a \leq c \leq b$
$\bullet b \leq a \leq c$
$\bullet b \leq c \leq a$
$\bullet c \leq a \leq b$
$\bullet c \leq b \leq a$

Vamos fazer apenas um caso para ilustrar. Suponhamos que $a \leq b \leq c$, então:

$$
\begin{aligned}
& \min \{a, \min \{b, c\}\}=\min \{a, b\}=a \\
& \min \{\min \{a, b\}, c\}=\min \{a, c\}=a
\end{aligned}
$$

Para concluir devemos verificar os outros cinco casos. 
Logo a aplicação acima é uma T-norma.

Note que, dados dois conjuntos fuzzy $A, B$ subconjuntos de $X$, para cada $x \in X$, podemos escrever

$$
u_{A \cap B}(x)=\min \left\{u_{A}(x), u_{B}(x)\right\}=T\left(u_{A}(x), u_{B}(x)\right) .
$$

Podemos então utilizar a T-norma para generalizar o conceito de interseção entre conjuntos fuzzy.

Exemplo 2.6 Aqui apresentamos outros exemplos de T-normas que são utilizadas para generalizar interseções entre conjuntos fuzzy.

1. $T(a, b)=a \cdot b$ - produto algébrico;

2. $T(a, b)=\max \{0, a+b-1\}$ - diferença limitada;

3. $T(a, b)=\left\{\begin{array}{l}a \text { se } b=1 \\ b \text { se } a=1 \\ 0 \text { caso contrário }\end{array} \quad\right.$ interseção drástica

Um resultado que relaciona alguns dos exemplos já apresentados de Tnorma é o seguinte:

Teorema 2.2 Seja $T_{M}$ a interseção drástica apresentada no Exemplo 2.6, item 3) e $T$ uma $T$-norma qualquer. Então dados $a, b \in[0,1]$,

$$
T_{M}(a, b) \leq T(a, b) \leq \min \{a, b\}
$$

\section{Dem.:}

Pelos axiomas 1 e 3 da definição de T-norma, temos:

$$
T(a, b) \leq T(a, 1)=a
$$

e pelo axioma 2 ,

$$
T(a, b)=T(b, a) \leq T(b, 1)=b .
$$

Logo,

$$
\begin{gathered}
T(a, b) \leq a \text { e } T(a, b) \leq b, \text { ou seja, } \\
T(a, b) \leq \min \{a, b\}
\end{gathered}
$$


Por outro lado, $T(a, 1)=a$ e $T(1, b)=b$. Como $T(a, b) \leq \min \{a, b\}$ e $T(a, b) \in[0,1]$

$$
T(a, 0)=T(0, b)=0 .
$$

Pelo axioma 3,

$$
T(a, b) \geq T(a, 0)=T(0, b)=0,
$$

ou seja, $T_{M}(a, b) \leq T(a, b) \leq \min \{a, b\}$.

Chamamos de T-norma idempotente aquela que $T(a, a)=a$, para todo $a \in[0,1]$. Com relação a essa T-norma, temos um resultado interessante:

Teorema 2.3 A T-norma do mínimo é a única idempotente.

\section{Dem.:}

É claro que $\min \{a, a\}=a, \forall a \in[0,1]$. Suponha que exista uma T-norma tal que $T(a, a)=a, \forall a \in[0,1]$. Então, para qualquer $a, b \in[0,1]$, se $a \leq b$ :

$$
a=T(a, a) \leq T(a, b) \leq T(a, 1)=a .
$$

Então $T(a, b)=a=\min \{a, b\}$.

Por outro lado, se $b \leq a$, temos:

$$
b=T(b, b) \leq T(a, b) \leq T(1, b)=b .
$$

$\mathrm{E} \operatorname{logo} T(a, b)=b=\min \{a, b\}$. Ou seja, $T(a, b)=\min \{a, b\}, \forall a, b \in[0,1]$.

\subsubsection{T-Conormas}

Como vimos, a definição de T-norma pode ser usada para generalizar a noção de interseção entre conjuntos fuzzy. O dual desta noção chama-se T-conorma, e é usada para generalizar a união entre conjuntos fuzzy.

Definição 2.2 Dizemos que uma aplicação $T_{c}:[0,1] \times[0,1] \rightarrow[0,1]$ é uma T-conorma se:

1. $T_{c}(0, a)=a, \forall a \in[0,1]$

2. $T_{c}(a, b)=T_{c}(b, a), \forall a, b \in[0,1]$

3. Se $a \leq c$ e $b \leq d$ então $T_{c}(a, b) \leq T_{c}(c, d)$ 
4. $T_{c}\left(a, T_{c}(b, c)\right)=T_{c}\left(T_{c}(a, b), c\right), \forall a, b, c \in[0,1]$

O primeiro exemplo a ser verificado é a união de conjuntos fuzzy, que segue a mesma linha de demonstração do exemplo da interseção, por isso apenas citaremos.

Exemplo 2.7 $T_{c}(a, b)=\max \{a, b\}, \forall a, b \in[0,1]$ é uma $T$-conorma.

Exemplo 2.8 Vamos apresentar outros exemplos:

1. $T_{c}(a, b)=a+b-a d i c ̧ a \tilde{o}$ algébrica;

2. $T_{c}(a, b)=\min \{1, a+b\}-$ adiçâo limitada;

3. $T_{c}(a, b)=\left\{\begin{array}{l}a \text { se } b=0 \\ b \text { se } a=0-\text { união drástica } \\ 1 \text { caso contrário }\end{array}\right.$

Resultados análogos aos da T-norma também valem para T-conorma.

Teorema 2.4 Seja $T_{M C}$ a união drástica apresentada no Exemplo 2.8 e $T_{c}$ uma T-conorma qualquer. Então dados $a, b \in[0,1]$,

$$
\max (a, b) \leq T_{c}(a, b) \leq T_{M C}(a, b) .
$$

Dem.:

Análoga à demonstração do Teorema 2.2.

Teorema 2.5 A T-conorma do máximo é a única idempotente.

Dem.:

Análoga à demonstração do Teorema 2.3.

\subsubsection{Generalização de Complementar de um Conjun- to Fuzzy}

Seja $A$ um conjunto fuzzy de $X, v(x)$ a função pertinência de $A$. Vamos denotar por $c v$ o complementar fuzzy de $A$ do tipo $c$. Então, $c v(x)$ será interpretado como o grau de não-pertinência de $x$ em $A$. 
Definição 2.3 Dizemos que uma aplicação $c:[0,1] \rightarrow[0,1]$ é um complementar fuzzy de um conjunto fuzzy $A$, se:

1. $c(0)=1$ e $c(1)=0$;

2. Para todo $a, b \in[0,1]$, se $a \leq b$ então $c(b) \leq c(a)$;

3. $c(c(a))=a, \forall a \in[0,1]$;

4. c é uma função contínua.

Teorema $2.6 c:[0,1] \rightarrow[0,1]$ como definida acima é uma função bijetora.

\section{Dem.:}

Para mostrar que é sobrejetora, basta observar que para todo $a \in[0,1]$, existe $b=c(a) \in[0,1]$ tal que $c(b)=c(c(a))=a$.

Agora é injetora pois, se $c\left(a_{1}\right)=c\left(a_{2}\right)$, então:

$$
a_{1}=c\left(c\left(a_{1}\right)\right)=c\left(c\left(a_{2}\right)\right)=a_{2} .
$$

Observe que para definir a função complementar fuzzy acima, bastava termos apresentado os itens 2 e 3 , como vamos demonstrar abaixo.

Teorema 2.7 Seja $c:[0,1] \rightarrow[0,1]$ satisfazendo os axiomas 2 e 3 acima, então c também satisfaz os axiomas 1 e 4 .

\section{Dem.:}

Como o domínio de $c$ é $[0,1]$, então $c(0) \leq 1$ e $c(1) \geq 0$. Pelo axioma 2, $c(0) \leq 1$, então $c(c(0)) \geq c(1)$ e por 3 ,

$$
0=c(c(0)) \geq c(1)
$$

logo $c(1)=0$. De novo aplicando $3,1=c(c(1))=c(0)$.

Para mostrar 4, basta observar que como é bijetora e monótona, então $c$ não pode ter pontos de descontinuidade, pois se $a_{o}$ é um ponto de descontinuidade de $c$,

$$
b_{o}=\lim _{a \rightarrow a_{o}^{-}} c(a)>c\left(a_{o}\right)
$$

e logo existe $b_{1} \in[0,1]$ tal que $b_{o}>b_{1}>c\left(a_{o}\right)$ e não existe $a_{1} \in[0,1]$ com $c\left(a_{1}\right)=b_{1}$, mas isso contradiz a bijetividade de $c$. Logo $c$ é contínua em $[0,1]$. 
Exemplo 2.9 Vamos apresentar dois exemplos de complementar fuzzy:

1. O exemplo clássico de complementar do conjunto fuzzy u é

$$
c_{s}(u(x))=1-u(x), \forall x \in X \text {. }
$$

2. Outro exemplo é o chamado classe de Sugeno. Dado o conjunto fuzzy $u$, o complementar fuzzy é dado por:

$$
\left.c_{\lambda}(u(x))=\frac{1-u(x)}{1+\lambda u(x)}, \forall x \in X, \text { onde } \lambda \in\right]-1, \infty[.
$$

Para cada valor de $\lambda$ obtemos um complementar fuzzy.

Para maiores referências sobre T-normas e T-conormas, ver [23].

\subsubsection{Combinação de Operações}

$\mathrm{Na}$ teoria clássica de conjuntos, as operações de interseção e união estão relacionadas com seus complementares, através das bem conhecidas leis de De Morgan:

$$
\begin{aligned}
& (A \cap B)^{\prime}=A^{\prime} \cup B^{\prime} \\
& (A \cup B)^{\prime}=A^{\prime} \cap B^{\prime}
\end{aligned}
$$

É desejável que estas relações sejam mantidas em conjuntos fuzzy, mesmo depois de generalizarmos os conceitos de interseção e união entre eles.

Definição 2.4 Dizemos que uma T-norma $T$ e uma $T$-conorma $T_{c}$ são duais com relação a um complementar fuzzy se:

$$
\begin{aligned}
& c(T(a, b))=T_{c}(c(a), c(b)) \\
& c\left(T_{c}(a, b)\right)=T(c(a), c(b))
\end{aligned}
$$

Estas expressões traduzem as leis de De Morgan para conjuntos fuzzy. Os exemplos que queremos salientar são os apresentados anteriormente em T-norma e T-conorma.

Exemplo 2.10 São duais com relação ao complementar fuzzy $c_{s}$ : 
1. $T(a, b)=\min \{a, b\}$ e $T_{c}(a, b)=\max \{a, b\}$;

2. $T(a, b)=a \cdot b$ e $T_{c}(a, b)=a+b$;

3. $T(a, b)=\max \{0, a+b-1\}$ e $T_{c}(a, b)=\min \{1, a+b\}$;

4. $T(a, b)=\left\{\begin{array}{l}a \text { se } b=1 \\ b \text { se } a=1 \\ 0 \text { caso contrário }\end{array} \quad\right.$ e $T_{c}(a, b)=\left\{\begin{array}{l}a \text { se } b=0 \\ b \text { se } a=0 \\ 1 \text { caso contrário }\end{array}\right.$

Os conceitos de T-normas e T-conormas serão ilustrados mais adiante na generalização do conceito de extensão de aplicações como a de Zadeh.

\subsection{Niveis de um Conjunto Fuzzy}

Observe que a noção de "pertinência" ou não de um elemento ao conjunto tem um papel diferente na teoria de conjuntos fuzzy.

No caso fuzzy, um elemento "pertence" a um conjunto fuzzy com certo grau. O que se faz é introduzir "cortes" ou níveis de um conjunto fuzzy. Por exemplo, seja $X$ um conjunto clássico, $u: X \rightarrow[0,1]$ a função pertinência de um conjunto fuzzy $u, \alpha, \beta \in[0,1]$ tais que, $0<\beta<\alpha<1$, podemos então que: $x$ pertence a $u$ com grau no mínimo $\alpha$ se $u(x) \geq \alpha$;

Vamos apresentar este conceito de modo mais formal. Daqui por diante, vamos considerar sempre $X=\mathbb{R}^{n}$.

Definição 2.5 Para cada $\alpha \in[0,1]$, chamamos de $\alpha$-nivel do conjunto fuzzy $u$ ao seguinte subconjunto do $\mathbb{R}^{n}$ :

$$
\left.\left.[u]^{\alpha}=\left\{x \in \mathbb{R}^{n} ; u(x) \geq \alpha\right\} \text { se } \alpha \in\right] 0,1\right]
$$

$e$

$$
[u]^{0}=\operatorname{supp} u=\overline{\left\{x \in \mathbb{R}^{n} ; u(x)>0\right\}},
$$

onde suppu é o suporte de $u$.

Isto é, $[u]^{\alpha}$ é formado pelos elementos que estão em um grau de pertinência não inferior a $\alpha$, e $[u]^{0}$ é o conjunto de elementos que estão pelo menos no fecho do conjunto fuzzy $u$. 
Exemplo 2.11 No caso do Exemplo 2.1, onde $u(x)=x^{2}$ se $x \in[0,1] e$ $u(x)=0$ caso contrário, para cada $\alpha \in[0,1]$, temos

$$
[u]^{\alpha}=[\sqrt{\alpha}, 1] .
$$

Exemplo 2.12 No caso do Exemplo 2.2,

$$
\left[u_{n}\right]^{\alpha}=[n(\alpha-1)+1,1]
$$

para cada $\alpha \in[0,1]$ e para cada $n \in \mathbb{N}$.

Em nosso trabalho vamos utilizar o espaço dos conjuntos fuzzy cujos $\alpha$ níveis são compactos e não vazios. Este conjunto é denotado por:

$$
\mathcal{F}\left(\mathbb{R}^{n}\right)=\left\{u: \mathbb{R}^{n} \rightarrow[0,1] ; \alpha-\text { níveis são compactos, não-vazios }\right\}
$$

Um resultado importante em $\mathcal{F}\left(\mathbb{R}^{n}\right)$ é o seguinte:

Proposição 2.1 Seja u um conjunto fuzzy. $u \in \mathcal{F}\left(\mathbb{R}^{n}\right)$ se e somente se,

1. $[u]^{0}$ é compacto;

2. $[u]^{1}$ é não-vazio;

3. $u$ é semicontínua superiormente.

Dem.:

Vamos supor que valem as três propriedades. Como, para cada $\alpha \in(0,1]$, $[u]^{\alpha} \subset[u]^{0}$ e $[u]^{0}$ é compacto, basta mostrar que $[u]^{\alpha}$ é fechado.

Lembremos que $u$ ser semicontínua superiormente significa que fixado $x_{0} \in \mathbb{R}^{n}$ e dado $\epsilon>0$, existe $\delta>0$ tal que se $\left|x-x_{0}\right|<\delta$ temos $u(x)<$ $\epsilon+u\left(x_{0}\right)$.

Então, seja o conjunto $A_{\alpha}=\left\{x \in \mathbb{R}^{n} ; u(x)<\alpha\right\}$. Fixado $a \in A_{\alpha}$, seja $0<\epsilon<\alpha-u(a)$. Então existe $\delta>0$ tal que se $|x-a|<\delta$, temos $u(x)<\epsilon+u(a)<\alpha-u(a)+u(a)=\alpha$, ou seja, $A_{\alpha}$ é aberto e como $[u]^{\alpha}$ é o complementar de $A_{\alpha}$, este é fechado. 
Seja agora $u \in \mathcal{F}\left(\mathbb{R}^{n}\right)$. Fixemos $a \in \mathbb{R}^{n}$ tal que $u(a)<1$. Dado $\epsilon>0$ e $u(a)+\epsilon<1$, temos que $a \in A_{u(a)+\epsilon}$, que é um conjunto aberto, então existe $\delta>0$ tal que para todo $x \in \mathbb{R}^{n}$ tal que $|x-a|<\delta$, temos $x \in A_{u(a)+\epsilon}$, ou seja, $u(x)<u(a)+\epsilon, \operatorname{logo} u$ é semicontínua superiormente.

Existe uma estrutura linear definida em $\mathcal{F}\left(\mathbb{R}^{n}\right)$, dada por:

$$
\begin{gathered}
(u+v)(x)=\sup _{y+z=x} \min \{u(y), v(z)\} \\
(\lambda u)(x)=\left\{\begin{array}{l}
u\left(\frac{x}{\lambda}\right) \text { se } \lambda \neq 0 \\
\chi_{\{0\}}, \text { se } \lambda=0
\end{array}\right.
\end{gathered}
$$

onde $\chi_{A}=\left\{\begin{array}{l}1 \text { se } x \in A \\ 0, \text { caso contrário }\end{array}\right.$

Exemplo 2.13 Sejam $X=\mathbb{R}$

$$
\begin{aligned}
& u(x)=\left\{\begin{array}{l}
1-x, \text { se } x \in[0,1] \\
0, \text { caso contrário }
\end{array}\right. \\
& v(x)=\left\{\begin{array}{l}
x, \text { se } x \in[0,1] \\
0, \text { caso contrário }
\end{array}\right.
\end{aligned}
$$

Então $(u+v)(x)$ é dado pelo gráfico abaixo e $2 u(x)$, pela expressão:

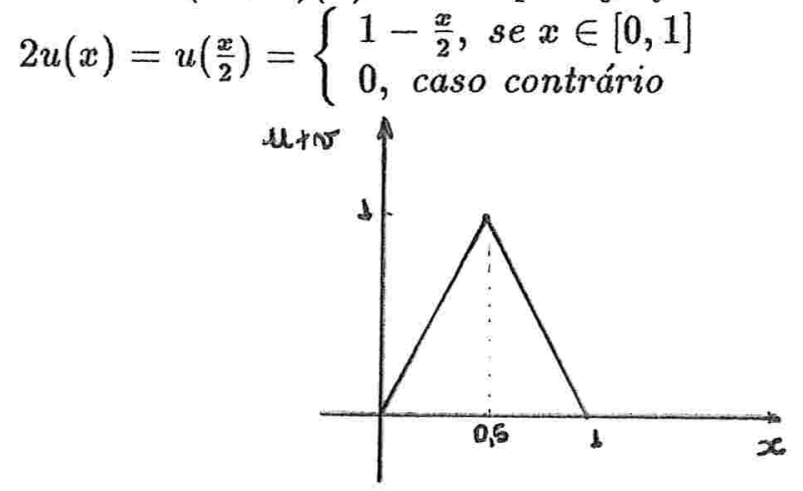

Observação 2.2 Para cada $\alpha \in[0,1]$

$$
[u+v]^{\alpha}=[u]^{\alpha}+[v]^{\alpha}
$$


e para $\lambda \in \mathbb{R}$,

$$
[\lambda u]^{\alpha}=\lambda[u]^{\alpha},
$$

onde $[u+v]^{\alpha}$ é a soma de conjuntos fuzzy e $[u]^{\alpha}+[v]^{\alpha}$ é a soma de Minkowski.

Considere o conjunto

$$
E^{n}=\left\{u \in \mathcal{F}\left(\mathbb{R}^{n}\right) ; u \text { é fuzzy convexo }\right\} .
$$

Dizemos que $u$ é fuzzy convexo se $u(\lambda x+(1-\lambda) y) \geq \min (u(x), u(y))$, para $\lambda \in[0,1]$. O conjunto $E^{n}$ é fechado em $\mathcal{F}\left(\mathbb{R}^{n}\right)$ na métrica $D$ que vamos apresentar adiante, ver [26] e, embora tenha uma estrutura linear, $E^{n}$ não é espaço vetorial.

Dentro deste contexto, temos um resultado muito importante (ver [26]), que caracteriza um conjunto fuzzy por uma família de conjuntos clássicos, a saber, seus $\alpha$-níveis.

Teorema 2.8 (Negoita, Ralescu) Seja $\aleph=\left\{A^{\alpha} ; 0 \leq \alpha \leq 1\right\}$ uma familia de conjuntos compactos e não-vazios do $\mathbb{R}^{n}$ tal que:

1. $\bigcup_{0<\alpha \leq 1} A^{\alpha} \subset A^{0}$,

2. $A^{\alpha_{2}} \subset A^{\alpha_{1}}$ para $0 \leq \alpha_{1} \leq \alpha_{2} \leq 1$,

3. Se $\alpha>0$ e $\alpha_{k} \nearrow \alpha, \operatorname{com} A^{\alpha}=\bigcap_{k \geq 1} A^{\alpha_{k}}$,

então existe um $u \in \mathcal{F}\left(\mathbb{R}^{n}\right)$ tal que $[u]^{\alpha}=A^{\alpha}$, para todo $0<\alpha \leq 1 e$

$$
[u]^{0}=\overline{\bigcup_{0<\alpha \leq 1} A^{\alpha}} \subset A^{0} .
$$

Em particular, se os conjuntos $A^{\alpha}$ forem convexos, para todo $0 \leq \alpha \leq 1$, então $u \in E^{n}$.

Dem.: Ver [22], porém, como indicação, para cada $x \in \mathbb{R}^{n}$, basta definir:

$$
u(x)=\sup \left\{\alpha \in[0,1] ; x \in A^{\alpha}\right\} .
$$




\subsection{Métricas no Espaço dos Conjuntos Fuzzy}

Vamos apresentar agora algumas métricas definidas no espaço dos conjuntos fuzzy e alguns resultados relativos a essas métricas. Antes vamos mostrar alguns resultados no espaço dos subconjuntos não vazios e compactos de um espaço métrico.

Dado um espaço métrico ( $\mathrm{Y}, \mathrm{d}$ ), e $A, B$ dois subconjuntos não-vazios e compactos de Y, a distância de Hausdorff entre $A$ e $B$ é dada por:

$$
h(A, B)=\inf \{\epsilon>0 ; B \subset N(A, \epsilon) \text { e } A \subset N(B, \epsilon)\}
$$

onde $N(A, \epsilon)=\{y \in \mathrm{Y} ; d(y, A)<\epsilon\}$ e $d(y, A)=\inf _{a \in A} d(y, a)$.

Sabe-se que $h$ é uma métrica no espaço dos subconjuntos não vazios e compactos de $\mathrm{Y}$ (ver [15]). Se $Y=\mathbb{R}^{n}$, denominamos este espaço de $\mathcal{Q}\left(\mathbb{R}^{n}\right)$. $\left(\mathcal{Q}\left(\mathbb{R}^{n}\right), h\right)$ é um espaço métrico completo e separável [26]. Uma definição equivalente para $h$ é:

$$
h(A, B)=\max \left\{\sup _{a \in A} d(a, B), \sup _{b \in B} d(b, A)\right\} .
$$

Para concluir isso, basta observar que:

Dado $r=\inf \{\epsilon>0 ; B \subset N(A, \epsilon)$ e $A \subset N(B, \epsilon)\}$, temos que $B \subset$ $N(A, r)$ e $A \subset N(B, r)$, ou seja, para todo $b \in B, d(b, A) \leq r$ e para todo $a \in A, d(a, B) \leq r$. Como $A$ e $B$ são compactos,

$$
\sup _{b \in B} d(b, A)=r \text { e } \sup _{a \in A} d(a, B)=r .
$$

Logo,

$$
\max \left\{\sup _{a \in A} d(a, B), \sup _{b \in B} d(b, A)\right\}=r .
$$

Por outro lado, se

$$
\max \left\{\sup _{a \in A} d(a, B), \sup _{b \in B} d(b, A)\right\}=r,
$$

então ou

$$
\sup _{a \in A} d(a, B)=r \text { ou } \sup _{b \in B} d(b, A)=r .
$$

Suponha que $\sup _{a \in A} d(a, B)=r$, então, para todo $a \in A, d(a, B) \leq r$, ou seja, $A \subset N(B, r)$, mas $\sup _{b \in B} d(b, A) \leq r$, logo para todo $b \in B, d(b, A) \leq r$, ou seja, $B \subset N(B, r)$. 
Suponha que exista $s<r$ tal que $A \subset N(B, s)$ e $B \subset N(A, s)$, então pela conclusão anterior, $\max \left\{\sup _{a \in A} d(a, B), \sup _{b \in B} d(b, A)\right\}=s$, o que é absurdo. Logo, $r=\inf \{\epsilon>0 ; B \subset N(A, \epsilon)$ e $A \subset N(B, \epsilon)\}$.

Proposição 2.2 Sejam $A, B$ e $C \in \mathcal{Q}\left(\mathbb{R}^{n}\right)$ e $A \subset B \subset C$, então

$$
h(B, X) \leq \max (h(A, X), h(C, X)),
$$

para todo $X \in \mathcal{Q}\left(\mathbb{R}^{n}\right)$.

Dem.: Vamos chamar $\rho(A, B)=\sup _{a \in A} d(a, B)$. Como $A \subset B \subset C$ e $A, B$ e $C$ são compactos, temos que:

$$
\begin{aligned}
& \rho(X, B) \leq \rho(X, A)+\rho(A, B)=\rho(X, A) \text { pois } A \subset B \\
& \rho(B, X) \leq \rho(B, C)+\rho(C, X)=\rho(C, X) \text { pois } B \subset C
\end{aligned}
$$

Logo, se $h(B, X) \geq h(A, X)$ então

$$
h(B, X)=\rho(B, X) \leq \rho(C, X) \leq h(C, X) ;
$$

e se $h(B, X) \geq h(C, X)$ então

$$
h(B, X)=\rho(B, X) \leq \rho(A, X) \leq h(A, X) .
$$

Logo,

$$
h(B, X) \leq \max (h(A, X), h(C, X)) .
$$

Definição 2.6 Uma seqüência $\left\{A_{p}\right\} \subset \mathcal{Q}\left(\mathbb{R}^{n}\right)$ converge para $A \subset \mathcal{Q}\left(\mathbb{R}^{n}\right)$ no sentido de Kuratowski, se

$$
\lim _{p \rightarrow \infty} \sup A_{p}=\lim _{p \rightarrow \infty} \inf A_{p}=A
$$

onde

$\lim _{p \rightarrow \infty} \sup A_{p}=\bigcap_{p=1}^{\infty} \overline{\left(\bigcup_{j=p}^{\infty} A_{j}\right)}=\left\{x \in \mathbb{R}^{n} ; x=\lim _{j \rightarrow \infty} x_{p_{j}} ; x_{p_{j}} \in A_{p_{j}}\right.$, subseq. de $\left.A_{p}\right\}$

e

$$
\lim _{p \rightarrow \infty} \inf A_{p}=\left\{x \in \mathbb{R}^{n} ; x=\lim _{p \rightarrow \infty} x_{p}, x_{p} \in A_{p}\right\}
$$


Teorema 2.9 Sejam $A, A_{p} \in \mathcal{Q}\left(\mathbb{R}^{n}\right)$. Então são equivalentes:

1. $\lim _{p \rightarrow \infty} h\left(A_{p}, A\right)=0$

2. $\left\{A_{p}\right\}$ converge para $A$ no sentido de Kuratowski e existe um compacto $K$ tal que $A \subset K$ e $A_{p} \subset K$, para todo $p$.

Dem.: Ver [15] .

Observando que para cada $\alpha \in[0,1]$ e $u \in \mathcal{F}\left(\mathbb{R}^{n}\right),[u]^{\alpha}$ são subconjuntos de $\mathcal{Q}\left(\mathbb{R}^{n}\right)$, podemos definir uma métrica em $\mathcal{F}\left(\mathbb{R}^{n}\right)$ dada por:

$$
D(u, v)=\sup _{0 \leq \alpha \leq 1} h\left([u]^{\alpha},[v]^{\alpha}\right)
$$

onde $u, v \in \mathcal{F}\left(\mathbb{R}^{n}\right)$

Desta maneira, $\left(\mathcal{F}\left(\mathbb{R}^{n}\right), D\right)$ é um espaço métrico, e conforme Puri-Ralescu [26], é completo, mas não separável [19].

Vamos ainda apresentar outras métricas em $\mathcal{F}\left(\mathbb{R}^{n}\right)$.

Dado $u \in \mathcal{F}\left(\mathbb{R}^{n}\right)$ e $d$ a métrica-produto em $\mathbb{R}^{n} \times[0,1]$ definida por:

$$
d((x, r),(y, s))=\max \{\|x-y\|,|r-s|\} .
$$

Chamamos de endográfico de $u$ o seguinte conjunto:

$$
\operatorname{end}(u)=\left\{(x, r) \in \mathbb{R}^{n} \times[0,1] ; u(x) \geq r\right\}
$$

e a partir daí define-se o sendográfico de $u$ por:

$$
\operatorname{send}(u)=\operatorname{end}(u) \cap\left([u]^{0} \times[0,1]\right) .
$$

Para ilustrar, seja $u: \mathbb{R}^{n} \rightarrow[0,1]$ dada abaixo. O sendográfico de $u$ está representado pela área hachurada da figura:

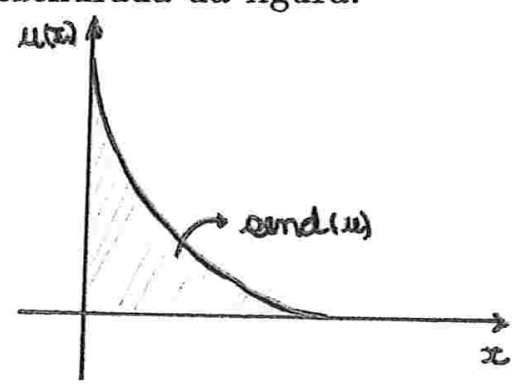

Agora então, seja a métrica $H$, ver [11], definida em $\mathcal{F}\left(\mathbb{R}^{n}\right)$ por:

$$
H(u, v)=h^{*}(\operatorname{send}(u), \operatorname{send}(v)),
$$


onde $h^{*}$ é a métrica de Hausdorff definida em $\mathbb{R}^{n} \times[0,1]$.

Conforme Kloeden [11], $\left(\mathcal{F}\left(\mathbb{R}^{n}\right), H\right)$ é um espaço métrico separável mas não completo. Outra referência para este assunto se encontra em [29].

A seguir vamos apresentar algumas convergências no espaço $\mathcal{F}\left(\mathbb{R}^{n}\right)$ e a relação entre elas.

\subsection{Convergências em $\mathcal{F}\left(\mathbb{R}^{n}\right)$}

Como já apresentamos seqüências em $\mathcal{F}\left(\mathbb{R}^{n}\right)$, vamos estudar alguns tipos de convergências.

Definição 2.7 Sejam $\left\{u_{n}\right\}$ uma seqüência em $\mathcal{F}\left(\mathbb{R}^{n}\right)$ e $u \in \mathcal{F}\left(\mathbb{R}^{n}\right)$.

Dizemos que $\left\{u_{n}\right\} D$-converge para $u$ se $D\left(u_{n}, u\right) \rightarrow 0$ quando $n \rightarrow \infty$.

Dizemos que $\left\{u_{n}\right\} L$-converge para $u$, ou converge em níveis para $u$ se para todo $\alpha \in] 0,1], h\left(\left[u_{n}\right]^{\alpha},[u]^{\alpha}\right) \longrightarrow 0$ quando $n \rightarrow \infty$.

Dizemos que $\left\{u_{n}\right\} H$-converge para $u$ se $H\left(u_{n}, u\right) \longrightarrow 0$ quando $n \rightarrow \infty$.

Teorema 2.10 São válidas as seguintes implicações:

1. D-convergência implica em L-convergência.

2. D-convergência implica em H-convergência.

Dem.:

A primeira implicação é óbvia. Vamos demonstrar a segunda.

Dado $\epsilon>0$ e $u, v \in \mathcal{F}\left(\mathbb{R}^{n}\right)$ tais que $D(u, v)<\epsilon$, vamos fixar um par $(x, \alpha) \in \operatorname{send}(u)$.

Se $\alpha>0$, então $x \in[u]^{\alpha}$. Como $h\left([u]^{\alpha},[v]^{\alpha}\right)<\epsilon$, existe um $y \in[v]^{\alpha}$ tal que $\|x-y\|<\epsilon$. Logo

$$
(x, \alpha) \in N(\operatorname{send}(v), \epsilon), \text { para }(y, \alpha) \in \operatorname{send}(v) .
$$

Se, por outro lado, $\alpha=0$, então $x \in[u]^{0}$. Vamos mostrar que $h\left([u]^{0},[v]^{0}\right)<$ $\epsilon$, pois poderemos encontrar um $y \in[v]^{0}$ tal que $\|x-y\|<\epsilon$, logo concluire$\operatorname{mos}$ que $(x, \alpha) \in N(\operatorname{send}(v), \epsilon)$.

Seja $\left\{\alpha_{n}\right\}$ uma seqüência decrescente de números reais convergindo para zero. Então, $\left\{[u]^{\alpha_{n}}\right\}$ é uma seqüência não decrescente de subconjuntos do 
conjunto compacto $[u]^{0}$. Logo, existe uma subseqüência $H$-convergente cujo limite pode ser expresso por:

$$
\bigcap_{n=1}^{\infty} \overline{\left(\bigcup_{m \geq n}[u]^{\alpha_{m}}\right)}
$$

Como $\alpha_{n} \rightarrow 0$ e $\left\{[u]^{\alpha_{n}}\right\}$ é não decrescente, vemos que o limite é $[u]^{0}$. Então,

$$
\lim h\left([u]^{\alpha_{n}},[u]^{0}\right)=0
$$

e de modo análogo para $v$.

Como $h$ é uma métrica, temos:

$$
h\left([u]^{0},[v]^{0}\right) \leq h\left([u]^{0},[u]^{\alpha_{n}}\right)+h\left([u]^{\alpha_{n}},[v]^{\alpha_{n}}\right)+h\left([v]^{\alpha_{n}},[v]^{0}\right)
$$

Passando o limite à direita de $\alpha$ indo para 0 ,

$$
h\left([u]^{0},[v]^{0}\right) \leq \lim _{\alpha \rightarrow 0_{+}} \sup _{\alpha>0} h\left([u]^{\alpha},[v]^{\alpha}\right)=D(u, v)<\epsilon
$$

Logo, $\operatorname{send}(v) \subset N(\operatorname{send}(u), \epsilon)$.

Observemos pelos exemplos que se seguem, que as recíprocas não são verdadeiras, ou seja, $L$-convergência ou $H$-convergência não implicam $D$ convergência.

\section{Exemplo 2.14 Seja}

$$
\begin{aligned}
& u(x)=\left\{\begin{array}{l}
1, \text { se } x \in[0,1] \\
0, \text { caso contrário }
\end{array}\right. \\
& u_{n}(x)=\left\{\begin{array}{l}
1+\frac{x-1}{n}, \text { se } x \in[0,1] \\
0, \text { caso contrário }
\end{array}\right.
\end{aligned}
$$

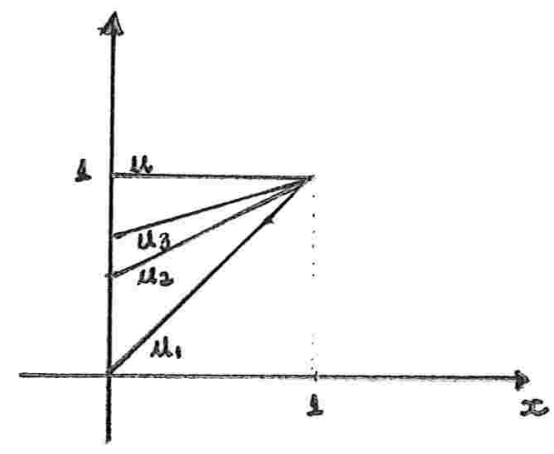

para $n \in \mathbb{N}^{*}$. 
Note que claramente $\left\{u_{n}\right\} H$-converge para $u$, logo:

$\lim _{n \rightarrow \infty} H\left(\operatorname{send}\left(u_{n}\right), \operatorname{send}(u)\right)=0, \operatorname{mas} h\left(\left[u_{n}\right]^{1},[u]^{1}\right)=1$, para todo $n \in \mathbb{N}^{*}$, ou seja, $\left\{u_{n}\right\}$ nâo converge em níveis e, portanto, não converge na métrica $D$.

Exemplo 2.15 Seja

$v(x)=\left\{\begin{array}{l}1, \text { se } x=0 \\ 0, \text { caso contrário }\end{array}\right.$
$v_{n}(x)=\left\{\begin{array}{l}(1-x)^{n}, \text { se } x \in[0,1] \\ 0, \text { caso contrário }\end{array}\right.$

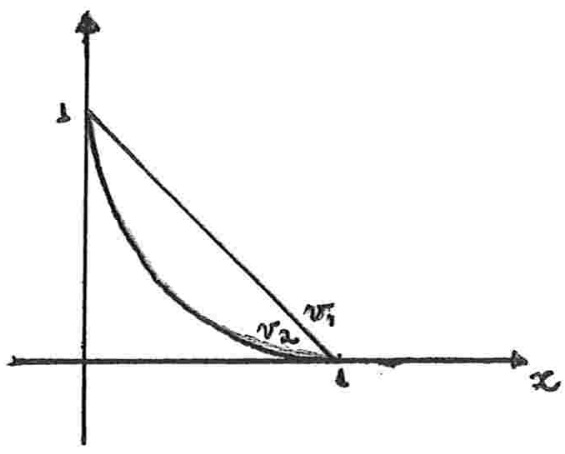

para $n \in \mathbb{N}^{*}$.

Como já vimos, $\left[v_{n}\right]^{\alpha}=\left[0,1-\alpha^{\frac{1}{n}}\right]$, logo $h\left([v]^{\alpha},\left[v_{n}\right]^{\alpha}\right)=1-\alpha^{\frac{1}{n}}$, para $\left.\left.\alpha \in\right] 0,1\right]$, portanto $\left\{v_{n}\right\}$ converge em níveis para $v$. Mas $h\left([v]^{0},\left[v_{n}\right]^{0}\right)=1$, para todo $n \in \mathbb{N}^{*}$, ou seja, $\left\{v_{n}\right\}$ não converge para v na métrica $D$.

Com o objetivo de reverter algumas implicações do Teorema 2.10 vamos nos restringir ao subespaço $\mathcal{F}^{*}\left(\mathbb{R}^{n}\right)$ de $\mathcal{F}\left(\mathbb{R}^{n}\right)$ :

$\mathcal{F}^{*}\left(\mathbb{R}^{n}\right)=\left\{u \in \mathcal{F}\left(\mathbb{R}^{n}\right) ; u\right.$ não tem pontos de máximos locais próprios e $[u]^{1}$ contém apenas um elemento $\}$, ou seja, para todo $x \in \mathbb{R}^{n}$ tal que $0<u(x)<1, x$ não é ponto de máximo local de $u: \mathbb{R}^{n} \rightarrow[0,1]$.

Note que para todo $u \in \mathcal{F}\left(\mathbb{R}^{n}\right)$, dizer que $u$ não tem pontos de máximos locais próprios é equivalente a

$$
[u]^{\alpha}=\overline{\left\{x \in \mathbb{R}^{n} ; u(x)>\alpha\right\}}
$$

para todo $\alpha \in] 0,1]$. 
Vamos dar um exemplo de $u \in \mathcal{F}^{*}\left(\mathbb{R}^{n}\right)$.

Exemplo 2.16 Seja $u: \mathbb{R} \rightarrow[0,1]$ dada por:

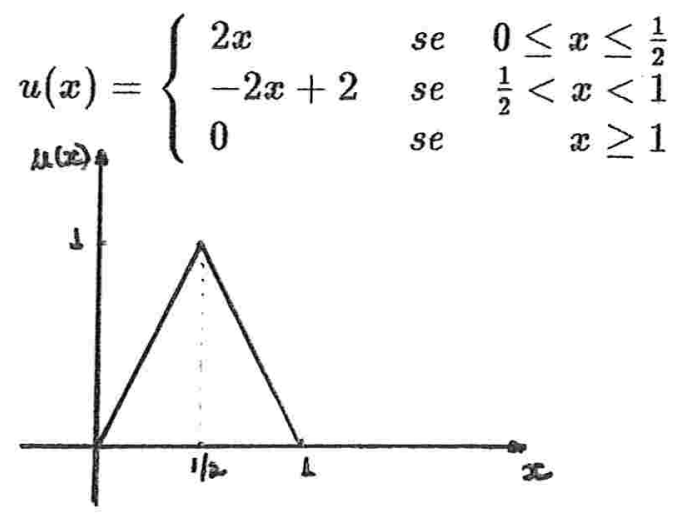

Exemplo 2.17 Seja $u: \mathbb{R} \rightarrow[0,1]$ dada por:

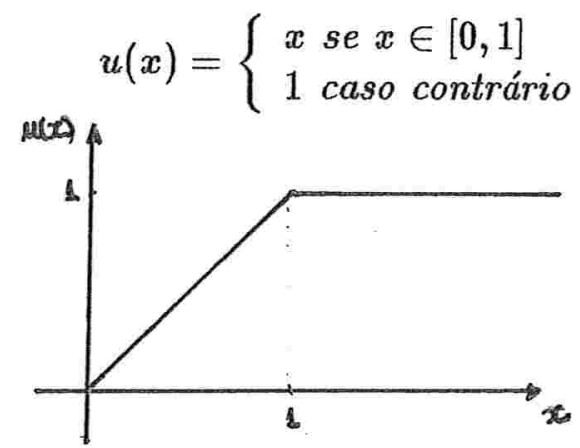

Embora $u$ não tenha pontos de máximos locais próprios, $u \notin \mathcal{F}^{*}\left(\mathbb{R}^{n}\right)$, pois $[u]^{1}$ tem mais que um ponto.

Proposição 2.3 Seja $u_{n}, u \in \mathcal{F}\left(\mathbb{R}^{n}\right)$, tal que u não tem pontos de máximos locais próprios. Então são equivalentes:

1. $u_{n}$ D-converge para $u$;

2. $u_{n} H$-converge para $u$ e $[u]^{1}=\liminf \left[u_{n}\right]^{1}$;

3. $u_{n} L$-converge para $u, \bigcup_{n=1}^{\infty}\left[u_{n}\right]^{0}$ é limitado e $\lim \sup \left[u_{n}\right]^{0} \subset[u]^{0}$. 


\section{Dem.:}

Ver Roman-Rojas [29] .

Observação 2.3 $\mathrm{Na}$ referência acima citada, os autores mostram que o fato de $u$ nâo ter pontos de máximos locais próprios é equivalente a u ter todos os seus $\alpha$-níveis contínuos em $\mathbb{R}^{n}$, ou seja, a aplicação $\alpha \mapsto[u]^{\alpha}$ é contínua nas métricas euclidiana e de Hausdorff, respectivamente.

Para maiores referências sobre convergências em $\mathcal{F}\left(\mathbb{R}^{n}\right)$, ver [27]. 


\section{Capítulo 3}

\section{A Extensão de Zadeh e Propriedades}

\subsection{O Princípio da Extensão}

Neste capítulo vamos tratar da "fuzificação de funções", ou seja, dada uma função $f: X \rightarrow Y$, como pode se estender esta função para o espaço de conjuntos fuzzy, $\mathcal{F}(X)$ e $\mathcal{F}(Y)$.

Da teoria clássica de conjuntos temos que, dada uma função $f: X \rightarrow Y$, considerando $\wp(X)$ e $\wp(Y)$ como sendo o conjunto das partes de $X$ e $Y$, respectivamente, podemos definir a extensão de $f$, denotada também por $f: \wp(X) \rightarrow \wp(Y)$, por

$$
f(A)=\{f(x) ; x \in A\}
$$

para cada $A \in \wp(X)$.

Assim também, pode-se estender $f^{-1}: \wp(Y) \rightarrow \wp(X)$, da seguinte maneira. Dado $B \in \wp(Y)$,

$$
f^{-1}(B)=\{x \in X ; f(x) \in B\}
$$

e, neste caso, $f^{-1}(B)$ pode ser vazio.

Agora, sejam $X$ um conjunto, $U, V$ subconjuntos de $X, u: U \rightarrow[0,1]$ e $v: V \rightarrow[0,1]$. Temos dois conjuntos fuzzy, que, como acima, podem ser estendidos de maneira clássica, ou seja, $u: \wp(U) \rightarrow \wp([0,1])$ e $v: \wp(V) \rightarrow$ $\wp([0,1])$.

Deste modo, se $K$ é um subconjunto de $U, u(K)$ é um subconjunto de $[0,1]$, portanto, podemos calcular $\sup u(K)$ e a função sup abaixo está bem 
definida:

$$
\begin{array}{cr}
\text { sup : } \wp(U) & \longrightarrow[0,1] \\
K & \mapsto \sup u(K)
\end{array}
$$

Agora, dados dois conjuntos $U$ e $V$, chama-se de Relação entre $U$ e $V$, um subconjunto qualquer de $U \times V$, ou seja, $R=\{(x, y) ; x \in U$ e $y \in V\}$. Podemos então induzir uma aplicação $R^{-1}: V \rightarrow \wp(U)$, dada por:

$$
R^{-1}(y)=\{x ;(x, y) \in R\} .
$$

Então, dado $u: U \rightarrow[0,1]$, temos:

$$
V \longrightarrow \wp(U) \longrightarrow \wp([0,1]) \rightarrow[0,1]
$$

Ou seja, a relação $R$ em $U \times V$ associada com a aplicação $u: U \rightarrow[0,1]$ dá origem a aplicação, que denotaremos por $\hat{R}=\sup \circ u \circ R^{-1}: V \rightarrow[0,1]$. Na verdade, se $R$ é uma função $f: U \rightarrow V$, então a extensão de $f$, que a cada $u: U \rightarrow[0,1]$ associa $\hat{f}(u): V \rightarrow[0,1]$, da seguinte maneira:

$$
\hat{f}(u)(x)=\left\{\begin{array}{lll}
\sup _{\tau \in f^{-1}(x)} u(\tau) & \text { se } & f^{-1}(x) \neq \emptyset \\
0 & \text { se } & f^{-1}(x)=\emptyset
\end{array}\right.
$$

Este é o Princípio da Extensão e $\hat{f}$ é chamada de Extensão de Zadeh da função $f: U \rightarrow V$. Para referências, ver [18].

Vamos apresentar alguns exemplos da extensão de Zadeh de algumas funções elementares.

Exemplo 3.1 Seja $f: \mathbb{R}^{n} \rightarrow \mathbb{R}^{n}$, onde $f(x)=c$, então,

$$
\hat{f}(u)(x)=\left\{\begin{array}{lll}
1 & \text { se } & x \in f^{-1}(c) \\
0 & \text { se } & x \notin f^{-1}(c)
\end{array}\right.
$$

ou seja, $\hat{f}(u)=\chi_{\{c\}}$

Exemplo 3.2 Seja $f: \mathbb{R}^{n} \rightarrow \mathbb{R}^{n}$, e $f(x)=x$, então,

$$
\hat{f}(u)(x)=\sup _{\tau \in\{x\}} u(\tau)=u(x)
$$

$\log o \hat{f}(u)=u$. 
Exemplo 3.3 Seja $f: \mathbb{R}^{n} \rightarrow \mathbb{R}^{n}, a \in \mathbb{R}^{*}$ e $f(x)=a x$, então,

$$
\hat{f}(u)(x)=\sup _{\tau \in\{x / a\}} u(\tau)=u(x / a)=a u(x)
$$

$\log o \hat{f}(u)=a u$.

Exemplo 3.4 Seja $f: \mathbb{R}^{n} \rightarrow \mathbb{R}^{n}, f(x)=A x+b, A \in M_{n}(\mathbb{R})$ e $b \in \mathbb{R}^{n}$, então,

$$
\hat{f}(u)(x)=\left\{\begin{array}{lc}
\sup _{A \tau+b=x} u(\tau) & \text { se } A \tau+b=x \text { tiver solução } \\
0 & \text { caso contrário }
\end{array}\right.
$$

Logo, $\hat{f}(u)(x)=u\left(A^{-1}(x-b)\right)$, se $A^{-1}$ existir.

Outro exemplo de extensão é o seguinte:

Exemplo 3.5 Sejam $u: U \rightarrow[0,1]$ e $v: V \rightarrow[0,1]$. u é um subconjunto fuzzy de $U$ e $v$ é um subconjunto fuzzy de $V$. Então $u \times v$ é um subconjunto fuzzy de $U \times V$, definido por:

$$
(u \times v)(x, y)=\min \{u(x), v(y)\}
$$

Em particular, se fizermos o produto de $u$ com o conjunto $V$, temos:

$$
\begin{array}{rrr}
(u \times V)(x, y) & = & \min \{u(x), I d(y)\} \\
& = & \min \{u(x), 1\} \\
& = & u(x)
\end{array}
$$

Neste caso, associamos um subconjunto de $U$ com um de $U \times V$. Este tipo de extensão é chamada de Extensão Cilíndrica

\subsection{Propriedades da Extensão}

Vamos demonstrar alguns resultados a seguir que apresentam as propriedades da extensão de Zadeh, a partir da função dada.

Teorema 3.1 [18] Seja $f: X \rightarrow Y$ uma função qualquer e u um subconjunto fuzzy de $X$. Então para todo $\alpha \in[0,1]$, temos:

$$
[\hat{f}(u)]^{\alpha} \supseteq f\left([u]^{\alpha}\right)
$$


Dem.: Se $y \in f\left([u]^{\alpha}\right)$, então existe $x_{o} \in[u]^{\alpha}$ tal que $y=f\left(x_{o}\right)$. Então:

$$
\hat{f}(u)(y)=\sup _{\tau \in f^{-1}(x)} u(\tau) \geq u\left(x_{o}\right) \geq \alpha,
$$

ou seja, $y \in[\hat{f}(u)]^{\alpha}$.

Para mostrar que $[\hat{f}(u)]^{\alpha} \neq f\left([u]^{\alpha}\right)$, em geral, veja o exemplo abaixo:

Exemplo 3.6 Seja $X=\mathbb{N}, Y=\{a, b\}$

$$
f(n)=\left\{\begin{array}{ccc}
a & \text { se } & n \leq 10 \\
b & \text { se } & n>10
\end{array}\right.
$$

e $u(n)=1-\frac{1}{n}$, para todo $n \in \mathbb{N}$. Então,

$$
\begin{aligned}
& \hat{f}(u)(a)=\sup _{n \in f^{-1}(a)} u(n)=\frac{9}{10} \\
& \hat{f}(u)(b)=\sup _{n \in f^{-1}(b)} u(n)=1
\end{aligned}
$$

Tomando $\alpha=1$, vemos que $[\hat{f}(u)]^{1}=\{b\}$, pois:

$$
\hat{f}(u)=\left\{\begin{array}{l}
1 \text { se } x=b \\
\frac{9}{10} \text { se } x=a \\
0 \text { caso contrário }
\end{array}\right.
$$

Mas $f\left([u]^{1}\right)=0$, pois $[u]^{1}=\emptyset$, logo $[\hat{f}(u)]^{1} \neq f\left([u]^{1}\right)$.

Mas restringindo $X=Y=\mathbb{R}^{n}$, que são os espaços que vamos estudar, temos alguns resultados interessantes, que apresentamos a seguir.

Teorema 3.2 (barros) [3] Seja $f: \mathbb{R}^{n} \rightarrow \mathbb{R}^{n}$ sobrejetora. Então

$$
[\hat{f}(u)]^{\alpha}=f\left([u]^{\alpha}\right)
$$

para todo $u: \mathbb{R}^{n} \rightarrow[0,1]$ e $0 \leq \alpha \leq 1$, se e somente se, $u$ atingir o máximo em $f^{-1}(x)$, para todo $x \in \mathbb{R}^{n}$.

\section{Dem.:}

Vamos supor que $[\hat{f}(u)]^{\alpha}=f\left([u]^{\alpha}\right)$, para todo $u \in \mathcal{F}\left(\mathbb{R}^{n}\right)$ e para todo $\alpha \in[0,1]$. Fixemos $x \in \mathbb{R}^{n}$ e suponha que $\sup _{\tau \in f^{-1}(x)} u(\tau)=s$, ou seja, existe $y$ tal que $u(y) \geq s$ e $x=f(y)$. Mas pela definição de $s$, temos que $u(y)=s$, ou seja, $u$ atinge seu máximo em $f^{-1}(x)$. 
Agora, se $u$ atinge seu máximo em $f^{-1}(x)$, vamos mostrar a igualdade:

Se $x \in[\hat{f}(u)]^{\alpha}$, então $\hat{f}(u)(x) \geq \alpha$, ou $\sup _{\tau \in f^{-1}(x)} u(\tau) \geq \alpha$. Mas, por hipótese, existe $y \in f^{-1}(x)$ tal que $u(y)=\sup _{\tau \in f^{-1}(x)} u(\tau) \geq \alpha$, logo $x \in$ $f\left([u]^{\alpha}\right)$.

Por outro lado, se $x \in f\left([u]^{\alpha}\right)$, existe $y \in[u]^{\alpha}$ tal que $x=f(y)$, ou seja, $u(y) \geq \alpha$. Daí,

$$
\hat{f}(u)(x)=\sup _{\tau \in f^{-1}(x)} u(\tau) \geq u(y) \geq \alpha,
$$

isto é, $x \in[\hat{f}(u)]^{\alpha}$.

Teorema 3.3 (Barros)[3] Se $f: \mathbb{R}^{n} \rightarrow \mathbb{R}^{n}$ é contínua então a extensão de Zadeh $\hat{f}: \mathcal{F}\left(\mathbb{R}^{n}\right) \rightarrow \mathcal{F}\left(\mathbb{R}^{n}\right)$ está bem definida e

$$
[\hat{f}(u)]^{\alpha}=f\left([u]^{\alpha}\right), \forall \alpha \in[0,1]
$$

\section{Dem.:}

Vamos mostrar que $[\hat{f}(u)]^{\alpha}=f\left([u]^{\alpha}\right), \forall \alpha \in[0,1]$.

$1^{\circ}$ Caso: $\alpha>0$

Seja $x \in[\hat{f}(u)]^{\alpha}$, então $\hat{f}(u)(x) \geq \alpha$, e portanto $f^{-1}(x) \neq \emptyset$ e logo $[u]^{o} \cap f^{-1}(x) \neq \emptyset$. Então,

$$
\hat{f}(u)(x)=\sup _{\tau \in f^{-1}(x)} u(\tau)=\sup _{\tau \in[u]^{\circ} \cap f^{-1}(x)} u(\tau) \geq \alpha
$$

Como $f$ é contínua, então $f^{-1}(x)$ é fechado e logo $[u]^{\circ} \cap f^{-1}(x)$ é compacto. Usando o fato de $u$ ser semicontínua superiormente e $[u]^{o} \cap f^{-1}(x)$ ser compacto, existe um resultado (Rudin pág. 195) que diz que existe $y \in[u]^{o} \cap f^{-1}(x)$ tal que

$$
\sup _{\tau \in[u]^{\circ} \cap f^{-1}(x)} u(\tau)=u(y)
$$

Logo, $f(y)=x$ e $u(y) \geq \alpha$, ou seja, $x \in f\left([u]^{\alpha}\right)$.

No caso $f\left([u]^{\alpha}\right) \subset\left[\hat{f}(u)^{\alpha}\right]$ segue do teorema 3.1. $2^{\circ}$ Caso: $\alpha=0$

Temos que $A=\{x ; \hat{f}(u)(x)>0\}=f\{x ; u(x)>0\}=f(B)$.

Se $x \in A$ então $\sup _{\tau \in f^{-1}(x)} u(\tau)>0$. Então existe $y$ tal que $f(y)=x$ e $u(y)>0$, ou seja, $x \in f(B)$.

Se $x \in f(B)$, então existe $y \in B$ com $x=f(y)$ e, logo, $\sup _{\tau \in f^{-1}(x)} u(\tau) \geq$ $u(y)>0$, ou seja, $x \in A$. 
Logo, como $f$ é contínua, $\bar{A}=\overline{f(B)} \supset f(\bar{B})$ e pela compacidade de $\bar{B}$, temos $\bar{A}=\overline{f(B)}=\overline{f(\bar{B})}=f(\bar{B})$. portanto, $[\hat{f}(u)]^{o}=f\left([u]^{o}\right)$.

Para provar que $\hat{f}$ está bem definida, ou seja, que $\hat{f}(u) \in \mathcal{F}\left(\mathbb{R}^{n}\right)$, precisamos mostrar que os $\alpha$-níveis $[\hat{f}(u)]^{\alpha}$ são não vazios e compactos, para todo $\alpha \in[0,1]$, mas isso é conseqüência do demonstrado acima.

Corolário 3.1 Se $f$ é contínua então $\hat{f}$ é monótona, ou seja,

$$
\hat{f}(u) \subseteq \hat{f}(v) \text { se } u \subseteq v
$$

onde $u \subseteq v$ significa $u(x) \leq v(x), \forall x \in \mathbb{R}^{n}$.

\section{Dem.:}

Basta mostrar que $[\hat{f}(u)]^{\alpha} \subset[\hat{f}(v)]^{\alpha}$, para todo $\alpha \in[0,1]$. Mas como, para cada $\alpha \in[0,1]$, temos $[u]^{\alpha} \subset[v]^{\alpha}$, então:

$$
[\hat{f}(u)]^{\alpha}=f\left([u]^{\alpha}\right) \subset f\left([v]^{\alpha}\right)=[\hat{f}(u)]^{\alpha}, \forall \alpha \in[0,1] .
$$

Outro resultado interessante precisa de um lema anterior.

Seja $K \subset \mathbb{R}^{n}$ um conjunto compacto.

Lema 3.1 Seja $f: \mathbb{R}^{n} \rightarrow \mathbb{R}^{n}$ uniformemente contínua. Então a aplicação $f: K \rightarrow f(K)$, definida em $\mathcal{Q}\left(\mathbb{R}^{n}\right)$ com a métrica de Hausdorff também é uniformemente contínua.

\section{Dem.:}

Dado $\epsilon>0$, seja $\delta>0$ tal que $\|x-y\|<\delta$ implique $\|f(x)-f(y)\|<\epsilon$, para todo $x, y \in \mathbb{R}^{n}$.

Sejam $K$ e $C$ compactos tais que $h(K, C)<\delta$. Então, para todo $x \in K$, $\inf _{y \in C}\|x-y\|<\delta$, se, e somente se, $\|x-\bar{y}\|<\delta$, para algum $\bar{y} \in C$, e portanto, $\|f(x)-f(\bar{y})\|<\epsilon$, e logo $\inf _{y \in C}\|f(x)-f(y)\|<\epsilon$, para todo $x \in K$. Ou ainda,

$$
\sup _{x \in K} \inf _{y \in C}\|f(x)-f(y)\|<\epsilon .
$$

De maneira análoga, concluímos que,

$$
\sup _{y \in C} \inf _{x \in K}\|f(x)-f(y)\|<\epsilon .
$$

E logo, $h(f(K), f(C))<\epsilon$. 
Teorema 3.4 (Barros) [3] Se $f: \mathbb{R}^{n} \rightarrow \mathbb{R}^{n}$ é uniformemente contínua então a extensão de Zadeh $\hat{f}: \mathcal{F}\left(\mathbb{R}^{n}\right) \rightarrow \mathcal{F}\left(\mathbb{R}^{n}\right)$ é uniformemente contínua na métrica $D$.

\section{Dem.:}

Para cada $\alpha \in[0,1]$, dado $\epsilon>0$, pelo lema anterior, existe $\delta_{\alpha}>$ 0 tal que se $h\left([u]^{\alpha},[v]^{\alpha}\right)<\delta_{\alpha}$, implica $h\left(f\left([u]^{\alpha}\right), f\left([v]^{\alpha}\right)\right)<\epsilon$. Mas se $D(u, v)<\delta$, temos $h\left([u]^{\alpha},[v]^{\alpha}\right)<\delta$, para todo $\alpha \in[0,1]$, o que implica $h\left([\hat{f}(u)]^{\alpha},[\hat{f}(v)]^{\alpha}\right)<\epsilon$, para todo $\alpha \in[0,1]$. Logo,

$$
\sup _{0 \leq \alpha \leq 1} h\left([\hat{f}(u)]^{\alpha},[\hat{f}(v)]^{\alpha}\right)<\epsilon
$$

ou seja, se $D(u, v)<\delta$, então $D(\hat{f}(u), \hat{f}(v))<\epsilon$.

Teorema 3.5 (Barros)[3] Sejam $f: \mathbb{R}^{n} \rightarrow \mathbb{R}^{n}$ e $\hat{f}: \mathcal{F}\left(\mathbb{R}^{n}\right) \rightarrow \mathcal{F}\left(\mathbb{R}^{n}\right) a$ extensão de Zadeh de $f$. Então $f$ é lipschitziana com constante $k$ se, $e$ somente se, $\hat{f}$ é lipschitziana com constante $k$, na métrica $D$.

\section{Dem.:}

Se $f$ é lipschitziana então é contínua, logo, os resultados anteriores para funções contínuas valem.

Note que

$$
D(\hat{f}(u), \hat{f}(v))=\sup _{0 \leq \alpha \leq 1} h\left([\hat{f}(u)]^{\alpha},[\hat{f}(v)]^{\alpha}\right)
$$

e

$$
\begin{gathered}
h\left([\hat{f}(u)]^{\alpha},[\hat{f}(v)]^{\alpha}\right)=\max \left\{\sup _{x \in[u]^{\alpha}} \inf _{y \in[v]^{\alpha}}\|f(x)-f(y)\|, \sup _{y \in[v]^{\alpha}} \inf _{x \in[u]^{\alpha}}\|f(x)-f(y)\|\right\} \\
\leq \max \left\{\sup _{x \in[u]^{\alpha}} \inf _{y \in[v]^{\alpha}} k\|x-y\|, \sup _{y \in[v]^{\alpha}} \inf _{x \in[u]^{\alpha}} k\|x-y\|\right\} \\
=k h\left([u]^{\alpha},[v]^{\alpha}\right) .
\end{gathered}
$$

Logo, $D(\hat{f}(u), \hat{f}(v)) \leq k D(u, v)$.

Por outro lado, como

$$
\left[\hat{f}\left(\chi_{\{x\}}\right)\right]^{\alpha}=f(x)
$$

para todo $x \in \mathbb{R}^{n}$ e $\alpha \in[0,1]$, temos,

$$
D\left(\hat{f}\left(\chi_{\{x\}}\right), \hat{f}\left(\chi_{\{y\}}\right)\right)=\|f(x)-f(y)\|,
$$


logo, se $D(\hat{f}(u), \hat{f}(v)) \leq k D(u, v)$, temos que para cada $x, y \in \mathbb{R}^{n}$,

$$
\|f(x)-f(y)\|=D\left(\hat{f}\left(\chi_{\{x\}}\right), \hat{f}\left(\chi_{\{y\}}\right)\right) \leq k D(u, v)=k\|x-y\| .
$$

Os resultados de continuidade da extensão de Zadeh que são apresentados abaixo, primeiramente foram demonstrados por Barros [3] na métrica $H$ e depois Roman-Flores estendeu para a métrica $D[30]$.

Teorema 3.6 (Barros) $f: \mathbb{R}^{n} \rightarrow \mathbb{R}^{n}$ é contínua se, e somente se, a extensão de Zadeh $\hat{f}: \mathcal{F}\left(\mathbb{R}^{n}\right) \rightarrow \mathcal{F}\left(\mathbb{R}^{n}\right)$ é contínua na métrica $H$.

Dem.: Ver Barros [3]

Teorema 3.7 Se $f: \mathbb{R}^{n} \rightarrow \mathbb{R}^{n}$ é contínua, então $\hat{f}: \mathcal{F}\left(\mathbb{R}^{n}\right) \rightarrow \mathcal{F}\left(\mathbb{R}^{n}\right)$ é contínua na métrica $D$.

Dem.: Ver Roman-Flores [30].

\subsection{Extensão usando T-Conorma e T-Norma}

Sejam $X$ um conjunto, $U, V$ subconjuntos de $X, u: U \rightarrow[0,1]$ e $v: V \rightarrow$ $[0,1]$, subconjuntos fuzzy de $X$.

Dados $s, t \in U$, podemos calcular $T_{c}(u(s), u(t))$, onde $T_{c}$ é T-conorma definida de $[0,1] \times[0,1]$ em $[0,1]$.

Como vimos na seção 3.1, podemos estender as funções $u: \wp(U) \rightarrow$ $\wp([0,1])$ da seguinte maneira,

$$
u(A)=\{u(x) ; x \in A\},
$$

para cada $A \in \wp(U)$,

Da mesma maneira, podemos estender $T_{c}: \wp([0,1]) \times \wp([0,1]) \rightarrow \wp([0,1])$.

Para calcularmos então a T-conorma, devemos ter:

$$
u \times u: \wp(U) \times \wp(U) \rightarrow \wp([0,1]) \times \wp([0,1]) .
$$

Dada a função $f: U \rightarrow V$ e a sua extensão $f: \wp(U) \rightarrow \wp(V)$ vimos na seção 3.1 , que podemos também definir a sua inversa dada por: $f^{-1}$ : $\wp(V) \rightarrow \wp(U)$.

Agora, seja também, a função 


$$
\begin{array}{cl}
\sup : \wp([0,1]) & \rightarrow[0,1] \\
X & \mapsto \sup X
\end{array}
$$

Então uma extensão $\tilde{f}$ usando T-conorma é dada pelo diagrama abaixo: $V \longrightarrow \wp(U) \longrightarrow \wp(U) \times \wp(U) \longrightarrow \wp([0,1]) \times \wp([0,1]) \longrightarrow \wp([0,1]) \longrightarrow[0,1]$ ou seja,

$$
\tilde{f}(u)=\sup \left(T_{c}\left(u\left(f^{-1}\right), u\left(f^{-1}\right)\right),\right.
$$

ou ainda, para cada $x \in X$,

$$
\tilde{f}(u)(x)=\left\{\begin{array}{lll}
\sup _{\tau \in f^{-1}(x)} T_{c}(u(\tau), u(\tau)) & \text { se } & f^{-1}(x) \neq \emptyset \\
0 & \text { se } & f^{-1}(x)=\emptyset
\end{array}\right.
$$

Observemos que se a T-conorma escolhida for a do máximo, então esta extensão coincide com a extensão de Zadeh, mas se não for, pelo Teorema 2.4, temos uma ordem parcial, ou seja, $\hat{f}(u) \leq \tilde{f}(u), \forall u \in \mathcal{F}(U)$, já que:

$$
\max (a, b) \leq T_{c}(a, b), \forall a, b \in[0,1] .
$$

Poderíamos, também, estar definindo uma extensão usando T-norma, onde $\breve{f}(u): V \rightarrow[0,1]$ seria dada por:

$$
\check{f}(u)(x)=\left\{\begin{array}{lll}
\sup _{\tau \in f^{-1}(x)} T(u(\tau), u(\tau)) & \text { se } & f^{-1}(x) \neq \emptyset \\
0 & \text { se } & f^{-1}(x)=\emptyset
\end{array}\right.
$$

Neste caso, $\check{f}(u) \leq \hat{f}(u), \forall u \in \mathcal{F}(U)$, já que:

$$
T(a, b) \leq \min (a, b), \forall a, b \in[0,1] .
$$

Vale observar que estas extensões não alteram as propriedades de continuidade, se as T-normas ou T-conormas escolhidas forem contínuas. 


\section{Capítulo 4}

\section{Sistemas Dinâmicos Discretos e Dissipatividade}

Neste capítulo, vamos destacar alguns conceitos de Sistemas Dinâmicos Discretos já conhecidos. Todos estes conceitos estão em Hale [14]. Nosso objetivo é, no próximo capítulo, estudar a suavidade assintótica e a dissipatividade das extensões de Zadeh de uma função contínua $f: \mathbb{R}^{n} \rightarrow \mathbb{R}^{n}$.

\subsection{Sistemas Dinâmicos Discretos}

Seja $(X, d)$ um espaço métrico completo.

Definição 4.1 Seja $f: X \rightarrow X$ uma aplicação contínua. Um sistema dinâmico discreto é um sistema iterativo dado por:

$$
x_{n+1}=f\left(x_{n}\right), \quad \operatorname{com} x_{o} \in X .
$$

Exemplo 4.1 Seja $f: \mathbb{R} \rightarrow \mathbb{R}$ dada por $f(x)=x^{2}+1$ e $x_{o}=0$.

Então, $x_{1}=1, x_{2}=2, x_{3}=5, x_{4}=26, \ldots$

Chamamos de órbita positiva que passa pelo ponto $x \in X$ o seguinte conjunto:

$$
\gamma^{+}(x)=\bigcup_{n \geq 0} f^{n}(x)
$$


onde $f^{n}=f \circ \cdots \circ f$ n-vezes, ou seja, é a composta de funções $f$.

E de órbita negativa :

$$
\gamma^{-}(x)=\bigcup_{n \geq 0} H(n, x)
$$

onde $H(n, x)=\left\{y \in X\right.$; existe uma seqüência $\left\{x_{j} ; j \in \mathbb{Z}_{-}\right\} ; x_{o}=x$ e $f\left(x_{j-1}\right)=x_{j}$, passando por $x$, tal que $x_{n}=y$, para algum $\left.n \in \mathbb{Z}_{-}\right\}$

A órbita completa é definida por $\gamma(x)=\gamma^{+}(x) \cup \gamma^{-}(x)$.

Observe que se $f$ não é injetora, $H(n, x)$ possui mais de uma seqüência. Se $B \subset X$, os conjuntos $\omega$-limite e $\alpha$-limite de $B$, respectivamente, são dados por:

$$
\begin{gathered}
\omega(B)=\bigcap_{n \geq 0} \overline{\left[\bigcup_{k \geq n} f^{k}(B)\right]} \\
\alpha(B)=\bigcap_{n \geq 0} \overline{\left[\bigcup_{k \geq n} H(k, B)\right]}
\end{gathered}
$$

Observe que podemos ter $\omega(B)=\emptyset$. Por exemplo, se $f: \mathbb{R} \rightarrow \mathbb{R}$ é tal que $f(x)=x^{2}$ e $B=\{2,3\}$.

Também, se $f(B) \subset B$, então $\omega(B)=\bigcap_{n>0} \overline{\left[f^{n}(B)\right]}$, pois, neste caso, $\bigcup_{k \geq n} f^{k}(B) \subset f^{n}(B)$, para todo $n \geq 0$.

Para um ponto $x \in X$ e $B=\{x\}$, o $w$-limite deste ponto passa a ser $\omega(x)=\left\{y \in X\right.$; existe uma seqüência $n_{j} \rightarrow \infty$ tal que $f^{n_{j}}(x) \rightarrow y$ quando $j \rightarrow \infty\}$

Definição $4.2 U m$ conjunto $S \subset X$ é dito invariante sob $f$ se para todo $x \in S$, existe uma órbita completa que passa por $x$ e $\gamma(x) \subset S$.

Exemplo 4.2 Seja $f: \mathbb{R} \rightarrow \mathbb{R}$ dada por $f(x)=x^{2}$.

$S=\{0\}$ é invariante pois $\gamma(0) \subset S$.

$S=\{0,1\}$ é invariante pois $\gamma(0)$ e $\gamma(1)$ estão contidos em $S$.

$[-1,1]$ é invariante, mas $] 1,2]$ não é invariante, pois $\gamma(2) \not \subset S$.

Lema 4.1 Sejam $f: X \rightarrow X$ e $S \subset X . S$ é invariante se, e somente se, $f(S)=S$. 


\section{Dem.:}

Suponha $S$ é invariante. Vamos mostrar que $S \subset f(S)$. Seja $x \in S$. Como, $\gamma(x) \subset S, f^{n}(x) \in S$, para todo $n \in \mathbb{N}$ e existe $y \in S$ tal que $x=f(y)$. Logo $x \in f(S)$. Mostrar que $f(S) \subset S$ é imediato.

Agora, seja $f(S)=S$ e $x \in S$, logo, $f(x) \in S$, e por indução sobre $n$, $f^{n}(x) \in S$, para todo $n \in \mathbb{N}$, ou seja, $\gamma^{+}(x) \subset S$. Tome então, $y_{o}$ tal que $x=f\left(y_{o}\right)$. Seja $y_{1} \in S$ tal que $y_{o}=f\left(y_{1}\right)$. Por indução sobre $n$, tem-se $y_{n}=f^{n}\left(y_{n-1}\right)$, ou seja, $\gamma(x) \subset S, S$ é invariante.

Definição 4.3 Sejam $f: X \rightarrow X$ e $A \subset X$. Um conjunto $A \subset X$ é dito atrator de um conjunto $B$ sob $f$ se para todo $\epsilon>0$, existe um $n_{o}=n_{o}(\epsilon, A, B)$ tal que $f^{n}(B) \subset N(A, \epsilon)$ para todo $n \geq n_{o}$; onde $N(A, \epsilon)$ é a vizinhança de centro $A$ e raio $\epsilon$.

Exemplo 4.3 Consideremos os exemplos abaixo:

1. em $\mathbb{R}$, seja $f(x)=\frac{x}{2}$.

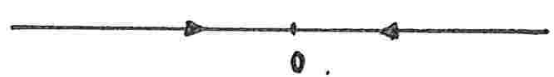

$\{0\}$ é atrator de $B$, para todo $B \subset \mathbb{R}$, compacto.

2. $e m \mathbb{R}$, seja

$$
f(x)=\left\{\begin{array}{lll}
\frac{x}{2} & \text { se } & x \geq 1 \text { ou } x<0 \\
2 x & \text { se } & x \in[0,1[
\end{array}\right.
$$

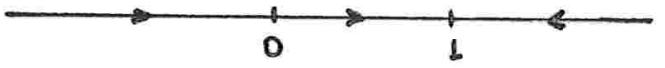

$[0,1]$ é atrator de $B$, para todo $B \subset \mathbb{R}$, compacto.

3. em $\mathbb{R}^{2}$, seja $f(x)=\frac{x}{2}$ :

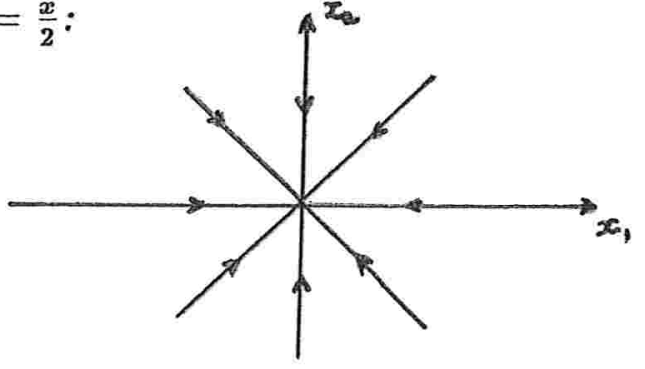

$\{0\}$ é atrator de $B$, para todo $B \subset \mathbb{R}^{2}$, compacto. 
Lema 4.2 (Hale) Se $\omega(B)$ é compacto e atrai $B$, então $\omega(B)$ é invariante.

\section{Dem.:}

Se $\omega(B)=\emptyset$, nada tem que se provar.

Suponhamos que $\omega(B) \neq \emptyset$. Observe que $f(\omega(B)) \subset \omega(B)$, pois $\omega(B)$ atrai $B$. Seja $y \in \omega(B)$, então existem seqüências $x_{j} \in B$, e $k_{j} \rightarrow \infty$, quando $j \rightarrow \infty$, tais que $f^{k_{j}}\left(x_{j}\right) \rightarrow y$ quando $j \rightarrow \infty$. Considere o conjunto $H=\left\{f^{k_{j}-1}\left(x_{j}\right) ; j \geq 1\right\}$. $H \subset \omega(B)$. Como $\omega(B)$ é compacto, existe uma subseqüência que converge para $z \in \omega(B)$. Ora, $f(z)=y \in f(\omega(B))$. Logo, $\omega(B)$ é invariante.

Lema 4.3 (Hale) Se $B$ é um subconjunto de $X$, não vazio e $\overline{\gamma^{+}(B)}$ é compacto, então $\omega(B)$ é não vazio, compacto, invariante e atrai $B$.

\section{Dem.:}

Segue da definição de

$$
\omega(B)=\bigcap_{n \geq \mathbf{0}} \overline{\left[\bigcup_{k \geq n} f^{k}(B)\right]},
$$

que $\omega(B)$ é não vazio e é compacto. Falta mostrar que $\omega(B)$ atrai $B$. Suponha que não, então existe $\epsilon>0$, uma seqüência de inteiros, $k_{j} \rightarrow \infty$ quando $j \rightarrow \infty$, e uma seqüência $y_{j} \in B$ tal que $d\left(f^{k_{j}}(B), \omega(B)\right)>\epsilon$ para $j=1,2,3, \cdots$

Como $\left\{f^{k_{j}}(B) ; j \geq 1\right\} \subset \overline{\gamma^{+}(B)}$ que é compacto, existe uma subseqüência convergente de $\left\{f^{k_{j}}\left(y_{j}\right)\right\}$. Como o limite desta seqüência pertence a $\omega(B)$, temos uma contradição.

Vamos agora definir alguns tipos de estabilidade.

Definição 4.4 Seja $f: X \rightarrow X$ contínua e $J$ invariante.

Diz-se que $J$ é estável se para toda vizinhança $V$ de $J$, existe uma vizinhança $U$ de $J$ tal que $f^{n}(U) \subset V$, para todo $n \geq 0$.

$J$ atrai pontos localmente se existe uma vizinhança $W$ de $J$ tal que $J$ atrai pontos de $W$.

$J$ é assintoticamente estável se $J$ é estável e atrai pontos localmente.

$J$ é uniformemente assintoticamente estável se $J$ é estável e atrai uma vizinhança de $J$.

Claramente, se um conjunto $J$ é uniformemente assintoticamente estável então $J$ é assintoticamente estável. O contrário não ocorre, a não ser em casos especiais, como por exemplo quando o espaço $X$ tem dimensão finita. Vamos ver alguns outros exemplos mais adiante. 


\subsection{Aplicações Assintoticamente Suaves}

Definição 4.5 Uma aplicação $f: X \rightarrow X$ contínua é assintoticamente suave se para todo subconjunto $B$ de $X$, não vazio, fechado e limitado, tal que $f(B) \subset B$, existe um subconjunto $J \subset B$ compacto tal que $J$ atrai $B$.

Observe que se $f: \mathbb{R}^{n} \rightarrow \mathbb{R}^{n}$, então toda função contínua é assintoticamente suave, bastando tomar $J=B$, já que todo fechado e limitado é compacto em um espaço de dimensão finita. Em um espaço de dimensão infinita o mesmo não acontece, veja o exemplo abaixo:

Exemplo 4.4 Considere $X=\ell_{2}(\mathbb{N})$, o espaço das seqüências cujas soma dos quadrados de seus termos é finita, e $f: X \rightarrow X$ dada por $f(x)=\frac{1}{2} x$ Seja $B=\left\{x \in \ell_{2}(\mathbb{N}) ;\|x\| \leq 1\right\}$

$B$ é fechado e limitado e não é compacto. Neste caso, $f$ não é assintoticamente suave.

Temos algumas classes de funções, que em um espaço de dimensão infinita têm resultados interessantes, como o abaixo:

Proposição 4.1 Seja $f: X \rightarrow X$ assintoticamente suave e $B \subset X, B \neq \emptyset$, limitado em $X$ tal que $\gamma^{+}(B)$ é limitado. Então $\omega(B)$ é não-vazio, compacto, invariante e $\omega(B)$ atrai $B$.

Dem.:

Como $f\left(\gamma^{+}(B)\right) \subset \gamma^{+}(B)$ e $f$ é contínua, temos que $f\left(\overline{\gamma^{+}(B)}\right) \subset \overline{\gamma^{+}(B)}$. Como $f$ é assintoticamente suave, existe um conjunto compacto $J \subset \overline{\gamma^{+}(B)}$ tal que $J$ atrai $B$, ou seja, existem seqüências $\epsilon_{j} \rightarrow 0 ; k_{j} \rightarrow \infty$ tais que $f^{n}(B) \subset N\left(J, \epsilon_{j}\right)$, para todo $n \geq k_{j}$. Isto implica que $\omega(B) \subset B$. Como $\omega(B)$ é fechado e $J$ é compacto, temos que $\omega(B)$ é compacto.

Falta mostrar que $\omega(B)$ atrai $B$. Suponha que não. Então existe $\epsilon>0$ e seqüências de inteiros $n_{j} \rightarrow \infty$ quando $j \rightarrow \infty$ e de pontos $y_{j} \in B$ tais que $f^{n}\left(y_{j}\right) \notin N(\omega(B), \epsilon)$. Como $J$ atrai $B$ e $J$ é compacto, podemos supor que $f^{n_{j}}\left(y_{j}\right) \rightarrow z \in J$. Mas então $z \in \omega(B)$, que é uma contradição. 
Exemplo 4.5 Seja $f: \mathbb{R}^{2} \rightarrow \mathbb{R}^{2}$ uma função dada por

$$
f(x, y)=\left(y+x\left(1-x^{2}-y^{2}\right),-x+y\left(1-x^{2}-y^{2}\right)\right)
$$

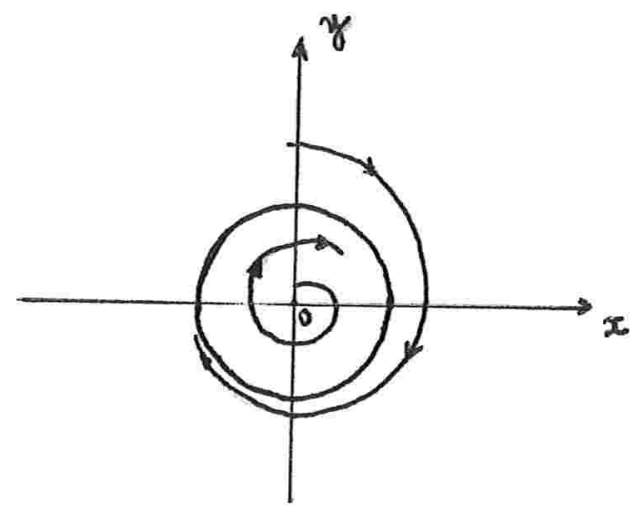

$\gamma^{+}\left(B_{1}(0)\right) \subset \overline{B_{1}(0)}$ e $\omega\left(B_{1}(0)\right)=\left\{x \in \mathbb{R}^{2} ;\|x\|=1\right\}$.

Outro resultado interessante é o seguinte:

Teorema 4.1 Se $f$ é assintoticamente suave e $J$ um conjunto compacto invariante contido em $X$, que atrai pontos localmente, então são equivalentes:

1. J é estável;

2. $J$ é uniformemente assintoticamente estável;

3. Existe uma vizinhança limitada $W$ de $J$ tal que $f(W) \subset W$ e $J$ atrai compactos de $W$.

\section{Dem.:}

Ver Hale [14], página 10.

Observe, pelo teorema anterior, que se $f$ é assintoticamente suave e $J$ é compacto e invariante, dizer que $J$ atrai pontos localmente é equivalente a dizer que $J$ atrai compactos localmente.

Vamos apresentar também alguns conceitos de dissipatividade. 


\subsection{Dissipatividade}

Definição 4.6 Seja $f: X \rightarrow X$ uma aplicação contínua em $X$.

Dizemos que $f$ é dissipativa por pontos em $X$ se existe um conjunto limitado $B$ em $X$ tal que $B$ atrai cada ponto de $X$.

$f$ é dita compacta dissipativa em $X$ se existe $B \subset X$ limitado tal que $B$ atrai cada conjunto compacto de $X$.

$f$ é localmente compacta dissipativa em $X$ se existe $B \subset X$ limitado tal que $B$ atrai uma vizinhança de cada conjunto compacto de $X$.

$f$ é limitada dissipativa em $X$ se existe $B \subset X$ limitado tal que $B$ atrai cada conjunto limitado de $X$.

Observe que se $f$ é compacta dissipativa, então $f$ é localmente compacta dissipativa e se $f$ é localmente compacta dissipativa, então $f$ é limitada dissipativa.

Seja $A \subset X$ um conjunto não-vazio. Dizemos que $A$ é maximal compacto invariante se $A$ é compacto, invariante e todo conjunto compacto invariante por $f$ está contido em $A$.

$A$ invariante é atrator global de $f$ se $A$ é maximal compacto invariante e atrai todo conjunto limitado $B$ de $X$.

Lema 4.4 (Hale) Se $X$ é um espaço de Banach e $J$ um conjunto compacto invariante que atrai compactos, então $J$ é conexo.

Dem.:

Seja $\overline{c o} J$ o fecho da envoltória convexa de $J$, então $\overline{c o} J$ é compacto conexo e $J$ atrai $\overline{c o} J$. Se $J$ não é conexo então existem conjuntos aberto $U, V$ com

$$
U \cap J \neq \emptyset ; V \cap J \neq \emptyset ; J \subset U \cup V \text { e } U \cap V=\emptyset
$$

Pela continuidade de $f, f^{n}(\overline{c o} J)$ é conexo, para todo $n \geq 0$.

Como $J \subset f^{n}(\overline{c o} J)$, para todo $n \geq 0$, temos que

$$
U \cap f^{n}(\overline{c o} J) \neq \emptyset ; V \cap f^{n}(\overline{c o} J) \neq \emptyset \text {, para todo } n \geq 0 .
$$

Mas $f^{n}(\overline{c o} J)$ é conexo, então existe um $x_{n} \in f^{n}(\overline{c o} J) \backslash(U \cup V)$, e por outro lado, como $J$ atrai $\overline{\left\{x_{n} ; n \geq 0\right\}}$ este conjunto tem que ser compacto.

Suponhamos então que $x_{n} \rightarrow x \in J$. Claramente $x \notin U \cup V$, o que é uma contradição. 
Teorema 4.2 (Hale) Seja $f: X \rightarrow X$ uma aplicação contínua tal que existe um conjunto $K$ não-vazio e compacto de $X$ que atrai conjuntos compactos de $X$ e seja $A=\bigcap_{n \geq 0} f^{n}(K)$, então:

1. A independe de $K$, ou seja, se existe $K_{1}$ nâo vazio compacto, subconjunto de $X$ que atrai conjuntos compactos de $X$, então $A=\bigcap_{n \geq 0} f^{n}\left(K_{1}\right)$, e, se $X$ é um espaço de Banach, A é conexo.

2. A é maximal, compacto e invariante.

3. A é estável e atrai conjuntos compactos de $X$.

Se além disso, $f$ é assintoticamente suave, então:

4. Para todo conjunto compacto $H$ em $X$, existe uma vizinhança $H_{1}$ de $H$ tal que $\gamma^{+}\left(H_{1}\right)$ é limitado e $A$ atrai $H_{1}$. Em particular, $A$ é uniformemente assintoticamente estável.

5. Se $C$ é um subconjunto de $X$ tal que $\gamma^{+}(C)$ é limitado, então $A$ atrai $C$.

\section{Dem.:}

1 e 2: Seja $H$ um compacto em $X$. Como $f^{n}(H) \rightarrow K$, o conjunto $\overline{\gamma^{+}(H)}$ é compacto e $\omega(B) \subset K$. Em particular, $\omega(K) \subset K$ e

$$
\omega(K)=\bigcap_{n>0} f^{n}(K)
$$

é não vazio, compacto e invariante, que atrai compactos de $X$. Pelo lema anterior, $\omega(K)$ é conexo.

Se $K_{1}$ é outro compacto que atrai compactos de $X$, então

$$
\omega\left(K_{1}\right) \subset \omega(K) \subset \omega\left(K_{1}\right),
$$

já que $\omega\left(K_{1}\right)$ atrai compactos. Logo, $A=\omega(K)$ independe de $K$.

3: Suponha que $A$ não seja estável. Então existe uma vizinhança $V$ de $A$ tal que para toda vizinhança $U$ de $A$, existe uma seqüência $\left\{x_{k}\right\} \subset U$ tal que $\left\{f^{n_{k}}\left(x_{k}\right) ; n_{k} \geq 0\right\} \not \subset V$.

Seja $U$ uma vizinhança compacta de $A$. Como $\left\{x_{k}\right\} \subset U$, existe uma subseqüência convergente $x_{k} \rightarrow x \in U$, e como $f$ é contínua para cada $n \geq 0$, 
$f^{n}\left(x_{k}\right) \rightarrow f^{n}(x)$. Seja $y=\lim _{n \rightarrow \infty} f^{n}(x) . y \in A$, e logo, $y \in V$, o que é uma contradição, logo $A$ é estável.

4: Como $A$ é estável, existe uma vizinhança $U$ de $A$ tal que $f(\bar{U}) \subset \bar{U}$. Como $A$ atrai compactos, para qualquer conjunto compacto $H$ de $X$, existe um inteiro $n_{0}$ que depende de $H$ e $U$, tal que $f^{n}(H) \subset U$, para $n \geq n_{0}$. E como $f$ é contínua e $H$ é compacto, existe uma vizinhança $H_{1}$ de $H$ tal que $f^{n_{0}}\left(H_{1}\right) \subset U$ e pelo fato de $f(U) \subset U$, segue que $f^{n}\left(H_{1}\right) \subset U$, para todo $n \geq n_{0}$. De $f$ ser assintoticamente suave, existe um conjunto compacto $K \subset U$ tal que $K$ atrai $U$. Como $A$ atrai $K$, então $A$ atrai $U$, e em particular, $A$ atrai $H_{1}$.

5: Seja $C \subset X$ com $\gamma^{+}(C)$ limitado. Como $f\left(\overline{\gamma^{+}(C)}\right) \subset \overline{\gamma^{+}(C)}$ e $f$ é assintoticamente suave, existe um conjunto compacto $K$ tal que $K$ atrai $\gamma^{+}(C)$. Então $A$ atrai $\gamma^{+}(C)$, e portanto $C$.

Observando o Teorema 4.2 e o Teorema 4.1, conclui-se que:

Corolário 4.1 Se $f: X \rightarrow X$ é assintoticamente suave, então são equivalentes:

1. Existe um conjunto compacto que atrai conjuntos compactos;

2. Existe um conjunto compacto que atrai vizinhanças de conjuntos compactos.

Lema 4.5 Se $f: X \rightarrow X$ é assintoticamente suave e é compacta dissipativa, então existe um conjunto compacto invariante que atrai compactos e vale as conclusões do teorema anterior.

Dem.:

Seja $C$ o conjunto limitado que atrai compactos em $X$. Tome

$$
B=\left\{x \in C ; f^{n}(x) \in C, n \geq 0\right\} \text {. }
$$

Então $f(\bar{B}) \subset \bar{B}$, logo existe um conjunto compacto $K$ que atrai $B$ e $B \subset C$ atrai compactos, logo $K$ atrai compactos.

O próximo teorema dá uma condição necessária para existência de um atrator. 
Teorema 4.3 Se $f: X \rightarrow X$ é assintoticamente suave, dissipativa por pontos e as órbitas de conjuntos limitados são limitadas, então existe um atrator global conexo.

\section{Dem.:}

Ver Hale [14].

No próximo capítulo aplicaremos alguns dos resultados apresentados aqui. Como o espaço $\mathcal{F}\left(\mathbb{R}^{n}\right)$ tem dimensão infinita, apesar de $f: \mathbb{R}^{n} \rightarrow \mathbb{R}^{n}$ contínua ser sempre assintoticamente suave, isto não acontece com a sua extensão de Zadeh $\hat{f}: \mathcal{F}\left(\mathbb{R}^{n}\right) \rightarrow \mathcal{F}\left(\mathbb{R}^{n}\right)$. Como veremos mais adiante, precisamos de hipóteses adicionais para garantir propriedades de suavidade assintótica e dissipatividade na extensão. 


\section{Capítulo 5}

\section{Dissipatividade em Sistemas Dinâmicos Fuzzy e Aplicações}

\subsection{Introdução}

Neste capítulo, apresentamos os nossos principais resultados. Nosso objetivo é estudar a extensão de Zadeh de uma função contínua em $\mathbb{R}^{n}$ e dizer quando ela é assintoticamente suave. Como vimos no capítulo anterior, a importância da função, que dá origem a um sistema dinâmico discreto, ser assintoticamente suave é que se um conjunto $J$ compacto e invariante atrai pontos localmente, então $J$ atrai compactos localmente, conforme o teorema 4.1.

Como também já vimos, só o fato de $f: \mathbb{R}^{n} \rightarrow \mathbb{R}^{n}$ ser contínua, já assegura que $f$ é assintoticamente suave, pois todo compacto do $\mathbb{R}^{n}$ é fechado e limitado, conforme [21], mas o caso muda quando estendemos para $\hat{f}: \mathcal{F}\left(\mathbb{R}^{n}\right) \rightarrow \mathcal{F}\left(\mathbb{R}^{n}\right)$ e é isso que queremos estudar. Na verdade o problema se resume em tomar um conjunto em $\mathcal{F}\left(\mathbb{R}^{n}\right)$ e dizer quando que ele é ou não compacto.

\subsection{Sobre Conjuntos Compactos em $\mathcal{F}\left(\mathbb{R}^{n}\right)$}

Nesta seção reunimos alguns resultados sobre o assunto. Kloeden e Diamond [11] apresentam uma caracterização de conjuntos compactos no espaço de conjuntos fuzzy cujos $\alpha$-níveis são convexos, porém não é exatamente este espaço que nos interessa, já que nosso espaço de interesse deve conter os 
conjuntos fuzzy cujos $\alpha$-níveis são apenas subconjuntos compactos do $\mathbb{R}^{n}$. Em Rojas et al. [31], é mostrado o seguinte:

Teorema 5.1 Seja $(X, d)$ um espaço métrico, $\mathcal{Q}(X)$ o espaço dos subconjuntos não-vazios e compactos de $X$ e

$$
\mathcal{F}(X)=\left\{u: X \rightarrow[0,1] ;[u]^{\alpha} \in \mathcal{Q}(X), \forall \alpha \in[0,1]\right\} .
$$

Então, $\mathcal{F}(X)$ é compacto se, e somente se $\operatorname{diam}(X)=0$, onde $\operatorname{diam}(X)$ é o diâmetro de $X$.

Assim, se $K \subset \mathbb{R}^{n}$ é compacto e $J \subset \mathcal{F}\left(\mathbb{R}^{n}\right)$ é tal que

$$
J=\left\{u \in \mathcal{F}\left(\mathbb{R}^{n}\right) ;[u]^{0} \subset K\right\},
$$

o conjunto $J$ é compacto se e só se $K$ tiver diâmetro zero! Veja o exemplo a seguir:

Exemplo 5.1 Considere $X=[0,1]$ com a métrica euclidiana. Neste caso, $X$ é um espaço métrico compacto (Lembrando que todo espaço compacto é se- parável [21], página 275).

Para cada $t \in[0,1]$ seja:

$$
u_{t}(x)=\left\{\begin{array}{llr}
t & \text { se } & x \in[0,1[ \\
1 & \text { se } & x=1
\end{array}\right.
$$

Observe que para todo $t,\left[u_{t}\right]^{\alpha}=[0,1]$, se $\alpha \leq t e\left[u_{t}\right]^{\alpha}=\{1\}$ se $\alpha>t$. Ou seja, $D\left(u_{t_{1}}, u_{t_{2}}\right)=1$, para todo $t_{1} \neq t_{2}$. Logo, $\mathcal{F}(X)$ não é separável, portanto não é compacto.

Mas apesar do conjunto

$$
J=\left\{u \in \mathcal{F}\left(\mathbb{R}^{n}\right) ;[u]^{0} \subset K, \text { onde } \mathrm{K} \text { é um compacto do } \mathbb{R}^{n}\right\},
$$

ser compacto se e somente se $K$ tiver diâmetro zero, ainda temos propriedades interessantes para este conjunto.

Lema 5.1 [3] Seja J como acima. J é um conjunto fechado e limitado do espaço métrico $\left(\mathcal{F}\left(\mathbb{R}^{n}\right), D\right)$. 


\section{Dem.:}

Para ver que $J$ é limitado, basta observar que a distância de $J$ ao conjunto fuzzy $\widehat{0}$ ( a função característica do conjunto $\{0\}$ ) é finita. De fato,

$$
\begin{array}{rrr}
D(\widehat{0}, J) & = & \inf _{u \in J} D(\widehat{0}, u) \\
& =\inf _{u \in J} \sup _{\alpha \in[0,1]} h\left(\{0\},[u]^{\alpha}\right) \\
& \leq & \sup _{\mathfrak{x} \in K} d(0, x)
\end{array}
$$

e esta última desigualdade é um número real $M<\infty$, pelo fato de $K$ ser um conjunto compacto do $\mathbb{R}^{n}$.

Para mostrar que $J$ é fechado, basta considerar uma seqüência $\left\{u_{n}\right\}$ em $J$ com limite $u$. Vamos mostrar que $u \in J$. Como $D\left(u_{n}, u\right) \longrightarrow 0$, pela definição da métrica $D$ temos que, em particular, $h\left(\left[u_{n}\right]^{\circ},[u]^{\circ}\right) \rightarrow 0$, e como $\left[u_{n}\right]^{o} \subset K$ e é uma seqüência de conjuntos compactos em $\mathcal{Q}\left(\mathbb{R}^{n}\right)$, segue que $[u]^{\circ} \subset K$, ou seja, $J$ é fechado, e isso completa a prova.

Temos um resultado que $X$ é um espaço métrico compacto se, e somente se é sequencialmente compacto([21] página 224). Como $\left(\mathcal{F}\left(\mathbb{R}^{n}\right), D\right)$ é um espaço métrico, mostrar que um conjunto $J \subset \mathcal{F}\left(\mathbb{R}^{n}\right)$ é compacto, basta mostrar que ele é sequencialmente compacto, ou seja, dada uma seqüência de pontos em $J$, pode-se extrair uma subseqüência convergente.

Antes de finalizar a seção vamos apresentar um lema que nos será útil mais adiante sobre a caracterização de bolas abertas no espaço dos conjuntos fuzzy $\mathcal{F}\left(\mathbb{R}^{n}\right)$.

Lema 5.2 (Barros)[3] Seja $\hat{X} \in \mathcal{F}\left(\mathbb{R}^{n}\right)$ a função característica do conjunto compacto $X \subset \mathbb{R}^{n}$. Então a bola aberta

$$
B(\hat{X}, r)=\left\{u \in \mathcal{F}\left(\mathbb{R}^{n}\right) ; D(\hat{X}, u)<r\right\}
$$

é o conjunto

$$
\left\{u \in \mathcal{F}\left(\mathbb{R}^{n}\right) ;[u]^{o} \subset B(X, r) \text { e } X \subset B\left([u]^{1}, r\right)\right\}
$$

\section{Dem.:}

Seja

$$
F=\left\{u \in \mathcal{F}\left(\mathbb{R}^{n}\right) ;[u]^{\circ} \subset B(X, r) \text { e } X \subset B\left([u]^{1}, r\right)\right\} .
$$

Se $u \in B(\hat{X}, r)$, então

$$
D(\hat{X}, u)=\sup _{0 \leq \alpha \leq 1} h\left(X,[u]^{\alpha}\right)<r
$$


ou

$$
\sup _{0 \leq \alpha \leq 1} \inf \left\{\epsilon ;[u]^{\alpha} \subset B(X, \epsilon) \text { e } X \subset B\left([u]^{\alpha}, \epsilon\right)\right\}<r
$$

que implica

$$
i(\alpha)=\inf \left\{\epsilon ;[u]^{\alpha} \subset B(X, \epsilon) \text { e } X \subset B\left([u]^{\alpha}, \epsilon\right)\right\}<r,
$$

para todo $\alpha \in[0,1]$. Então, $[u]^{\alpha} \subset B(X, r)$ e $X \subset B\left([u]^{\alpha}, r\right)$, para todo $\alpha \in[0,1]$. Em particular, temos $[u]^{\circ} \subset B(X, r)$ e $X \subset B\left([u]^{1}, r\right), \operatorname{logo} u \in F$, ou seja, $B(\hat{X}, r) \subset F$.

Se $u \in F$, então $[u]^{o} \subset B(X, r)$ e $X \subset B\left([u]^{1}, r\right)$, o que implica que $[u]^{\alpha} \subset[u]^{o} \subset B(X, r)$ e $X \subset B\left([u]^{1}, r\right) \subset B\left([u]^{\alpha}, r\right)$, para todo $\alpha \in[0,1]$. Ou seja, $[u]^{\alpha} \subset B(X, r)$ e $X \subset B\left([u]^{\alpha}, r\right)$, para todo $\alpha \in[0,1]$. Como $X$ e $[u]^{\alpha}$ são compactos, $B(X, r)$ e $B\left([u]^{\alpha}, r\right)$ são abertos e temos que $i(\alpha)<r$, para todo $\alpha \in[0,1]$. Agora,

$$
i(\alpha) \leq \max \{i(0), i(1)\}<r
$$

para todo $\alpha \in[0,1]$, usando a Proposição 2.2, já que

$$
i(\alpha)=h\left([u]^{\alpha}, X\right) \text { e }[u]^{1} \subset[u]^{\alpha} \subset[u]^{o} .
$$

Finalmente,

$$
\sup _{\mathbf{0} \leq \alpha \leq 1} i(\alpha)<r \text {, ou seja, } u \in B(\hat{X}, r) .
$$

Corolário 5.1 Se $X=\left\{x_{o}\right\}$ for unitário, entâo

$$
B(\hat{X}, r)=\left\{u \in \mathcal{F}\left(\mathbb{R}^{n}\right) ;[u]^{\circ} \subset B\left(x_{o}, r\right)\right\}
$$

e, da definição de $D$,

$$
D(\hat{X}, u)=\sup _{x \in[u]^{\circ}}\left\|x-x_{o}\right\| .
$$

Na próxima seção, reunimos alguns resultados interessantes e conhecidos sobre sistemas dinâmicos fuzzy. 


\subsection{Alguns Resultados sobre Sistemas Dinâmicos Fuzzy}

Seja $f: \mathbb{R}^{n} \rightarrow \mathbb{R}^{n}$ uma função contínua e

$$
x_{n+1}=f\left(x_{n}\right)
$$

um Sistema Dinâmico Discreto.

Considerando $\hat{f}: \mathcal{F}\left(\mathbb{R}^{n}\right) \rightarrow \mathcal{F}\left(\mathbb{R}^{n}\right)$ a extensão de Zadeh de $f$, dizemos que o sistema abaixo é o Sistema Fuzzy associado ao Sistema Discreto 5.1.

$$
u_{n+1}=\hat{f}\left(u_{n}\right)
$$

Teorema 5.2 (Barros)[3] Suponha que $x_{n}$ e $u_{n}$ sejam soluções dos sistemas 5.1 e 5.2 acima, que passam por $x_{o}$ e $u_{o}$ respectivamente, e que $u_{o}\left(x_{o}\right)=1$. Então, $u_{n}\left(x_{n}\right)=1$, para todo $n \geq 0$.

Dem.:

Temos a igualdade:

$$
u_{n+1}\left(x_{n+1}\right)=\hat{f}\left(u_{n}\right)\left(x_{n+1}\right)=\sup _{x_{n+1}=f(\tau)} u_{n}(\tau) \geq u_{n}\left(x_{n}\right)
$$

decorrente do fato de $x_{n}$ e $u_{n}$ serem soluções. Então,

$$
u_{n+1}\left(x_{n+1}\right) \geq u_{n}\left(x_{n}\right) \geq \cdots \geq u_{o}\left(x_{o}\right)=1
$$

Definição 5.1 Seja $F: \mathcal{F}\left(\mathbb{R}^{n}\right) \rightarrow \mathcal{F}\left(\mathbb{R}^{n}\right)$ uma aplicação. Dizemos que $\bar{u} \in$ $\mathcal{F}\left(\mathbb{R}^{n}\right)$ é um ponto fixo de $F$ se $F(\bar{u})=\bar{u}$.

Observe que $F(\bar{u})=\bar{u}$ se e somente se,

$$
[F(\bar{u})]^{\alpha}=[\bar{u}]^{\alpha},
$$

para todo $\alpha \in[0,1]$.

Teorema 5.3 (Barros)[3] Seja $f: \mathbb{R}^{n} \rightarrow \mathbb{R}^{n}$ contínua e $A \subset \mathbb{R}^{n}$, onde $A$ é um conjunto compacto. A é invariante se e somente se a função característica $\chi_{A}$ é ponto fixo da extensão de Zadeh $\hat{f}$. 


\section{Dem.:}

Segue do teorema 3.3 e do lema 4.1 que,

$$
\left[\hat{f}\left(\chi_{A}\right)\right]^{\alpha}=f\left(\left[\chi_{A}\right]^{\alpha}\right)=f(A)=A=\left[\chi_{A}\right]^{\alpha}, \forall \alpha \in[0,1] .
$$

Definição 5.2 Dada $F: \mathcal{F}\left(\mathbb{R}^{n}\right) \rightarrow \mathcal{F}\left(\mathbb{R}^{n}\right)$ e $\bar{u} \in \mathcal{F}\left(\mathbb{R}^{n}\right)$ um ponto fixo de $F$.

Dizemos que $\bar{u}$ é estável se para todo $\epsilon>0$ existir $\delta>0$ tal que, para todo u para o qual $\sup _{0 \leq \alpha \leq 1} h\left([u]^{\alpha},[\bar{u}]^{\alpha}\right)<\delta$, as iteradas por u satisfazem

$$
\sup _{0 \leq \alpha \leq 1} h\left(\left[F^{n}(u)\right]^{\alpha},[\bar{u}]^{\alpha}\right)<\epsilon, \text { para todo } n \geq 0 .
$$

Dizemos que $\bar{u}$ é assintoticamente estável se for estável $e$

$$
\lim _{n \rightarrow \infty} \sup _{0 \leq \alpha \leq 1} h\left(\left[F^{n}(u)\right]^{\alpha},[\bar{u}]^{\alpha}\right)=0
$$

desde que $\sup _{0 \leq \alpha \leq 1} h\left([u]^{\alpha},[\bar{u}]^{\alpha}\right)<r$.

Teorema 5.4 (Barros) [3] Seja $f: \mathbb{R}^{n} \rightarrow \mathbb{R}^{n}$ contínua com $f(\bar{x})=\bar{x}$ e $\hat{f}$ sua extensão de Zadeh. Então:

a) $\chi_{\{\bar{x}\}}$ é estável para o sistema 5.2 se, e somente se, $\bar{x}$ é estável para o sistema 5.1;

b) $\chi_{\{\bar{x}\}}$ é assintoticamente estável para o sistema 5.2 se, e somente se, $\bar{x}$ é assintoticamente estável para o sistema 5.1.

\section{Dem.:}

a) Por hipótese, dado $\epsilon>0$, existe $\delta>0$ tal que se $\|x-\bar{x}\|<\delta$ então $\left\|f^{n}(x)-\bar{x}\right\|<\epsilon$ para todo $n \geq 0$. Se $D\left(u, \chi_{\{\bar{x}\}}\right)<\delta$, então

$$
\sup _{x \in[u]^{\circ}}\|x-\bar{x}\|<\delta, \text { que implica }\left\|f^{n}(x)-\bar{x}\right\|<\epsilon,
$$

para todo $x \in[u]^{o} \subset B(\bar{x}, \delta)$. Logo,

$$
\sup _{x \in[u]^{\circ}}\left\|f^{n}(x)-\bar{x}\right\|<\epsilon
$$

ou

$$
\sup _{y \in f^{n}\left([u]^{\circ}\right)}\|y-\bar{x}\|<\epsilon
$$

ou ainda,

$$
\sup _{y \in\left[\hat{f}^{n}(u)\right]^{\circ}}\|y-\bar{x}\|<\epsilon
$$


pois $f$ é contínua.

Logo, pelo Corolário 5.1,

$$
D\left(\hat{f}^{n}(u), \chi_{\{\bar{x}\}}\right)<\epsilon .
$$

Para mostrar a estabilidade de $\bar{x}$, a partir da estabilidade de $\chi_{\{\bar{x}\}}$, basta observar que

$$
\|x-\bar{x}\|=D\left(\chi_{\{x\}}, \chi_{\{\bar{x}\}}\right)<\epsilon
$$

$\mathrm{e}$

$$
\hat{f}^{n}\left(\chi_{\{x\}}\right)=\chi_{\left\{f^{n}(x)\right\}} .
$$

b) Por hipótese, existe $r>0$ tal que

$$
\|x-\bar{x}\|<r \text { então } \lim _{n \rightarrow \infty}\left\|f^{n}(x)-\bar{x}\right\|=0 .
$$

Suponha que $D\left(u, \chi_{\{\bar{x}\}}\right)<r$, pelo Corolário 5.1 basta provar que para cada $\epsilon>0$ existe um $N>0$ tal que se $n>N$ então $f^{n}\left([u]^{o}\right) \subset B(\bar{x}, \epsilon)$.

Dado $\epsilon>0$, existe $\delta>0$ tal que se $\|x-\bar{x}\|<\delta$ então $\left\|f^{n}(x)-\bar{x}\right\|<\epsilon$, para cada $n>0$, pela estabilidade de $\bar{x}$.

Agora, para cada $x \in[u]^{\circ}$, existe um natural $n(x)$ tal que $f^{n(x)} \in B(\bar{x}, \delta)$, já que o ponto fixo $\bar{x}$ atrai cada ponto em $B(\bar{x}, r)$. Usando a continuidade de $f$ podemos escolher uma vizinhança aberta $V_{x}$ de $x$ tal que $f^{n(x)}\left(V_{x}\right) \subset B(\bar{x}, \delta)$. Fazendo isso para todos os pontos do conjunto compacto $[u]^{\circ}$, temos uma cobertura aberta de $[u]^{\circ}$ por $V_{x}$. Extraímos uma subcobertura finita $\left\{V_{x_{1}}, \cdots, V_{x_{k}}\right\}$ correspondendo aos pontos $x_{1}, \cdots, x_{k}$ em $[u]^{o}$. Note que $f^{n(x)+n}\left(V_{x}\right) \subset B(\bar{x}, \epsilon)$, para cada $n>0$.

Então, seja $N=\max \left\{n\left(x_{1}\right), \cdots, n\left(x_{k}\right)\right\}$, para cada $x \in[u]^{\circ}$ e $n>0$, $f^{N+n}(x) \in B(\bar{x}, \epsilon)$, já que $x \in V_{x_{i}}$, para algum $i \in\{1, \cdots, k\}$. Logo, $f^{N+n}\left([u]^{o}\right) \subset B(\bar{x}, \epsilon)$. A outra implicação é imediata, como no item a).

Corolário 5.2 Seja $f: \mathbb{R}^{n} \rightarrow \mathbb{R}^{n}$, de classe $C^{1}$ e $f(\bar{x})=\bar{x}$. Sendo $\lambda_{i}$ os autovalores de $f^{\prime}(\bar{x})$, então:

a) Se $\left|\lambda_{i}\right|<1$, para todo $i, \chi_{\{\bar{x}\}}$ é assintoticamente estável para 5.2;

b) $\mathrm{Se}\left|\lambda_{i}\right|>1$, para algum $i, \chi_{\{\bar{x}\}}$ é instável para 5.2.

\subsection{O Problema da Suavidade Assintótica em $\mathcal{F}\left(\mathbb{R}^{n}\right)$}

Nosso interesse em caracterizar conjuntos compactos está relacionado com o estudo da suavidade assintótica da extensão de Zadeh de uma função 
$f: \mathbb{R}^{n} \rightarrow \mathbb{R}^{n}$ contínua. A seguir listaremos alguns resultados que serão apresentados em uma seqüência de lemas.

Seja $f: \mathbb{R}^{n} \rightarrow \mathbb{R}^{n}$ uma função contínua e $\hat{f}: \mathcal{F}\left(\mathbb{R}^{n}\right) \rightarrow \mathcal{F}\left(\mathbb{R}^{n}\right)$ sua extensão de Zadeh, que, de acordo com o Teorema 3.7 é contínua.

Observação 5.1 Sejam $f$ e $\hat{f}$ como acima e $B \subset \mathcal{F}\left(\mathbb{R}^{n}\right)$, não vazio, fechado e limitado, tal que $\hat{f}(B) \subset B$. Para cada $u \in \hat{f}(B)$ existe $v \in B$ tal que $\hat{f}(v)=u$ e, para cada $\alpha \in[0,1]$ temos:

$$
[u]^{\alpha}=[\hat{f}(v)]^{\alpha}=f\left([v]^{\alpha}\right)
$$

O caminho que escolhemos para estudar a suavidade assintótica da $\hat{f}$ foi observando os $\alpha$-níveis já que são subconjuntos compactos do $\mathbb{R}^{n}$ e conhecemos muitas de suas propriedades.

A seguir, todos os lemas se referem a $f$ e $\hat{f}$ definidas acima e ao conjunto $B \subset \mathcal{F}\left(\mathbb{R}^{n}\right)$ também citado acima.

Defina

$$
B_{\alpha}=\overline{\bigcup_{u \in B}[u]^{\alpha}} \subset \mathbb{R}^{n}
$$

Lema 5.3 Seja $B_{\alpha}$ como acima. Existe um conjunto compacto $K \subset \mathbb{R}^{n}$ tal que $[u]^{\circ} \subset K$, para todo $u \in B$. Assim, $B_{\alpha}$ é limitado, para cada $\alpha \in[0,1]$.

\section{Dem.:}

Fixemos $x \in \mathbb{R}^{n}$ e vamos denotar por $\hat{x}$ a função característica do conjunto $\{x\}$. Como $B$ é limitado, existe um $r>0$ tal que $B$ está contido na bola de centro $\hat{x}$ e raio $r$. Em outras palavras, para cada $u \in B, D(\hat{x}, u) \leq r$. E como vimos acima,

$$
D(\hat{x}, u)=\sup _{0 \leq \alpha \leq 1} \inf \left\{\varepsilon ;[u]^{\alpha} \subset B_{\varepsilon}(\{x\}) \text { e }\{x\} \subset B_{\varepsilon}\left([u]^{\alpha}\right)\right\}<r .
$$

Em particular, para todo $\alpha \in[0,1],[u]^{\alpha} \subset B_{r}(\{x\})$, ou ainda, $[u]^{o} \subset B_{r}(\{x\})=K$, para todo $u \in B$, e isto prova o lema.

Lema 5.4 Considere $B_{\alpha}$ como no lema anterior. Então, para cada $\alpha \in[0,1], B_{\alpha}$ é fechado, limitado e satisfaz $f\left(B_{\alpha}\right) \subset B_{\alpha}$, e logo, existe um conjunto compacto $J_{\alpha} \subset B_{\alpha}$ que atrai $B_{\alpha}$. 
Dem.:

O único fato que precisa ser provado é que $f\left(B_{\alpha}\right) \subset B_{\alpha}$, pois o restante segue imediatamente das definições e do fato de que toda transformação contínua em $\mathbb{R}^{n}$ é assintoticamente suave, o que garante que existe $J_{\alpha} \subset B_{\alpha}$ que atrai $B_{\alpha}$, para cada $\alpha \in[0,1]$.

Seja $x \in B_{\alpha}$. Como $B_{\alpha}$ é fechado e limitado em $\mathbb{R}^{n}$, existe uma seqüência $x_{n} \rightarrow x, \operatorname{com} x_{n} \in\left[u_{n}\right]^{\alpha}$, para cada $n \in \mathbb{N}$ e $\left\{u_{n}\right\}$ uma seqüência em $B$. Como, $\hat{f}(B) \subset B$ e $\hat{f}\left(u_{n}\right) \in B$, então

$$
f\left(x_{n}\right) \in f\left(\left[u_{n}\right]^{\alpha}\right)=\left[\hat{f}\left(u_{n}\right)\right]^{\alpha} \subset B_{\alpha} .
$$

Como $f$ é contínua e $B_{\alpha}$ é compacto, $f\left(x_{n}\right)$ converge para $f(x)$ e $f(x) \in B_{\alpha}$, e isto completa a prova.

O fato de estarmos trabalhando com os conjuntos $B_{\alpha}$, que são compactos no $\mathbb{R}^{n}$ e $f: \mathbb{R}^{n} \rightarrow \mathbb{R}^{n}$ ser contínua, portanto assintoticamente suave, juntamente com o Lema anterior, que afirma que para cada $\alpha \in[0,1]$, temos $f\left(B_{\alpha}\right) \subset B_{\alpha}$, podemos aplicar a Proposição 4.1 que afirma que se $f$ é assintoticamente suave e $\gamma^{+}\left(B_{\alpha}\right)$ é limitado, então $\omega\left(B_{\alpha}\right)$ é não-vazio, compacto, invariante e $\omega\left(B_{\alpha}\right)$ atrai $B_{\alpha}$. E, de novo, por $f\left(B_{\alpha}\right) \subset B_{\alpha}$, temos que $\omega\left(B_{\alpha}\right) \subset B_{\alpha}$. Então podemos escolher os $J_{\alpha}=\omega\left(B_{\alpha}\right)$, para cada $\alpha \in[0,1]$, e com isso podemos utilizar algumas propriedades já conhecidas dos $\omega$-limites e apresentadas no capítulo 4 . Observe que:

Lema 5.5 Para $0 \leq \alpha_{1} \leq \alpha_{2} \leq 1$ temos $J_{\alpha_{2}} \subset J_{\alpha_{1}}$.

Dem.:

Como $B_{\alpha_{2}} \subset B_{\alpha_{1}}$ e $f\left(B_{\alpha i}\right) \subset B_{\alpha_{i}}$, para $i=1,2$, segue que o $\omega$-limite pode ser escrito assim:

$$
J_{\alpha_{2}}=\omega\left(B_{\alpha_{2}}\right)=\bigcap_{n \geq 0} f^{n}\left(B_{\alpha_{2}}\right) \subset \bigcap_{n \geq 0} f^{n}\left(B_{\alpha_{1}}\right)=J_{\alpha_{1}}
$$

e aí segue o resultado.

Seja $K=\omega(B)$ e $K_{\alpha}=\overline{\bigcup_{u \in K}[u]^{\alpha}}$. Observe a relação de igualdade que existe entre os $J_{\alpha}$ 's acima e os $K_{\alpha}$ 's.

Teorema 5.5 Suponha $\hat{f}: \mathcal{F}\left(\mathbb{R}^{n}\right) \longrightarrow \mathcal{F}\left(\mathbb{R}^{n}\right)$ assintoticamente suave, $B$ um subconjunto não vazio, fechado, limitado de $\mathcal{F}\left(\mathbb{R}^{n}\right)$, tal que $\hat{f}(B) \subset B$ e sejam $K_{\alpha}$ e $J_{\alpha}$ como acima. Então para cada $\alpha \in[0,1]$ temos $K_{\alpha}=J_{\alpha}$. 


\section{Dem.:}

Primeiro vamos mostrar que $K_{\alpha} \subset B_{\alpha}$ :

Seja $x \in K_{\alpha}$. Então existe $u \in \omega(B)$ tal que $x \in[u]^{\alpha}$ mas $\omega(B) \subset B$, pois $\hat{f}(B) \subset B$, logo $u \in B$, ou seja, $x \in B_{\alpha}$.

Agora, como $J_{\alpha}$ atrai $B_{\alpha}$ e $K_{\alpha} \subset B_{\alpha}$ para cada $\alpha \in[0,1]$, segue que $J_{\alpha}$ atrai $K_{\alpha}$, ou seja, para cada $\epsilon>0$, existe $n_{\alpha}$ tal que

$$
f^{n}\left(K_{\alpha}\right) \subset N\left(J_{\alpha}, \epsilon\right),
$$

para cada $n \geq n_{\alpha}$, e usando o fato de que $f\left(K_{\alpha}\right) \subset K_{\alpha}, K_{\alpha} \subset N\left(J_{\alpha}, \epsilon\right)$. Como $\epsilon$ é arbitrário, segue que $K_{\alpha} \subset J_{\alpha}$.

Falta provar que $J_{\alpha} \subset K_{\alpha}$. Pela Proposição $4.1, K$ atrai $B$, então, dado $\epsilon>0$ existe $n_{o}$ tal que para $n \geq n_{o}, \hat{f}^{n}(B) \subset N(K, \epsilon)$, isto implica que para cada $\alpha \in[0,1], f^{n}\left(B_{\alpha}\right) \subset N^{*}\left(K_{\alpha}, \epsilon\right)$, onde

$$
N^{*}\left(K_{\alpha}, \epsilon\right)=\left\{x \in \mathbb{R}^{n}: d\left(x, K_{\alpha}\right) \leq \epsilon\right\} .
$$

Segue da definição de $J_{\alpha}$ que

$$
J_{\alpha} \subset \bigcap_{n \geq n_{o}} f^{n}\left(B_{\alpha}\right) \subset N^{*}\left(K_{\alpha}, \varepsilon\right)
$$

E vale para todo $\varepsilon>0$, donde segue o resultado.

Seja

$$
J=\left\{u \in B:[u]^{o} \subset J_{o}\right\} .
$$

Como já vimos no lema $5.1, J$ é fechado, limitado e pelo Teorema 5.1, $J$ é compacto se e somente se $J_{o}$ tiver diâmetro zero. Mas uma condição necessária para que $\hat{f}$ seja assintoticamente suave é dada pelo resultado abaixo:

Teorema 5.6 Se $\hat{f}: \mathcal{F}\left(\mathbb{R}^{n}\right) \longrightarrow \mathcal{F}\left(\mathbb{R}^{n}\right)$ é assintoticamente suave, entẫo $J$ é não vazio, para todo $B$ não vazio, fechado limitado tal que $\hat{f}(B) \subset B$.

Dem.:

Seja $B$ não vazio, fechado e limitado tal que $\hat{f}(B) \subset B$. Como, por hipótese, $\hat{f}$ é assintoticamente suave, pela Proposição $4.1, \omega(B)$ é não vazio, compacto, invariante e atrai $B$.

Vamos chamar $K=\omega(B)$ e seja

$$
K_{o}=\overline{\bigcup_{u \in K}[u]^{a}} .
$$


Com relação a $K_{o}$, temos:

a) $K_{o}$ é um conjunto compacto, pois é o fecho de um conjunto limitado, já que $K \subset B$

b) $K_{o}$ está contido em $B_{o}$.

De fato, pois se $x \in K_{o}$, então existe $u \in K$ tal que $x \in[u]^{o}$, mas $K=\omega(B)$ e como $\hat{f}(B) \subset B$, temos que $\omega(B)=K \subset B$. Logo, $u \in B$, ou seja, $x \in B_{o}$.

c) $K_{o}$ é um conjunto invariante sob $f$ (isto é, $f\left(K_{o}\right)=K_{o}$ ).

De fato, se $x \in K_{o}$ então sabemos que $x$ é limite de uma seqüência $\left\{x_{n}\right\}$ tal que $x_{n} \in\left[u_{n}\right]^{o}$ e $u_{n} \in \omega(B)$. Então segue que: $f(x)=\lim _{n \rightarrow \infty} f\left(x_{n}\right)$. Agora,

$$
f\left(x_{n}\right) \in f\left(\left[u_{n}\right]^{\circ}\right)=\left[\hat{f}\left(u_{n}\right)\right]^{o} \in K_{o}
$$

isto mostra que $f\left(K_{o}\right) \subset K_{o}$.

Para provar que $f\left(K_{o}\right) \supset K_{o}$, vamos repetir o processo, tomando $x \in K_{o}$ e $x_{n}$ como acima. Agora, como $K$ é invariante sob $\hat{f}$, temos que

$$
u_{n}=\hat{f}\left(v_{n}\right), \text { onde } v_{n} \in K \text {. }
$$

Então, para cada $n \geq 0$ temos $x_{n}=f\left(y_{n}\right)$, onde $y_{n} \in\left[v_{n}\right]^{\circ} \subset K_{o}$. Escolhendo uma subseqüência $y_{n_{j}}$ de $y_{n}$, se necessário, temos $y=\lim _{j \rightarrow \infty} y_{n_{j}}$. Por continuidade de $f$, segue que $x=f(y)$. Logo $K_{o}$ é um conjunto invariante de $f$.

Agora, $J_{o}$ atrai $B_{o}$ e também atrai $K_{o}$. Ou seja, para cada $\varepsilon>0$, existe um $n_{o}$ tal que para cada $n \geq n_{o}, f^{n}\left(K_{o}\right) \subset N\left(J_{o}, \varepsilon\right)$, ou, usando a invariância de $K_{o}, K_{o} \subset N\left(J_{o}, \varepsilon\right)$. Pelo fato de $\varepsilon$ ser arbitrário, segue que $K_{o} \subset J_{o}$, e logo, $J \neq \emptyset$.

Corolário 5.3 Se $J=\emptyset$ então $\hat{f}$ não é assintoticamente suave.

\subsection{Um Exemplo em $\mathcal{F}^{*}\left(\mathbb{R}^{n}\right)$}

Como já definimos anteriormente, $\mathcal{F}^{*}\left(\mathbb{R}^{n}\right)$ é o espaço dos conjuntos fuzzy $u \in \mathcal{F}\left(\mathbb{R}^{n}\right)$ tais que $u$ não tem pontos de máximos locais próprios e $[u]^{1}$ é um conjunto unitário. Dada uma aplicação contínua $f: \mathbb{R}^{n} \rightarrow \mathbb{R}^{n}$ com a extensão $\hat{f}$ restrita ao espaço $\mathcal{F}^{*}\left(\mathbb{R}^{n}\right)$, vamos mostrar que, neste caso, $\hat{f}$ é assintoticamente suave, se todos os conjuntos $B \subset \mathcal{F}\left(\mathbb{R}^{n}\right)$ não vazios, limitados e fechados com $\hat{f}(B)$ subset $B$ tiverem seus $w$-limites não vazios. 
[4].

Primeiro, precisamos do seguinte resultado, cuja prova se encontra em

Proposição 5.1 (Barros)[3] Se $f: \mathbb{R}^{n} \rightarrow \mathbb{R}^{n}$ é uma aplicação contínua, então:

(i) $\hat{f}\left(\mathcal{F}^{*}\left(\mathbb{R}^{n}\right)\right) \subset \mathcal{F}^{*}\left(\mathbb{R}^{n}\right)$;

(ii) $\hat{f}$ restrita ao espaço $\mathcal{F}^{*}\left(\mathbb{R}^{n}\right)$ é contínua na métrica $D$.

Um resultado sobre seqüências se faz necessário:

Teorema 5.7 Sejam $\left\{A_{n}\right\}$ e $\left\{B_{n}\right\}$ duas seqüências de conjuntos compactos, tal que $A_{n} \subset B_{n}$ para todo $n \in \mathbb{N}, B_{n} \longrightarrow B$ e $A_{n_{j}} \longrightarrow$ A na métrica de Hausdorff. Então $A \subset B$.

\section{Dem.:}

Pelo Teorema $2.9, \lim _{p \rightarrow \infty} h\left(A_{p}, A\right)=0$, se e somente se $A_{p}$ converge para $A$ no sentido de Kuratowski , onde limsup $\operatorname{sum}_{p \rightarrow \infty} A_{p}=\bigcap_{p=1}^{\infty}\left(\overline{\bigcup_{j=p}^{\infty} A_{j}}\right)$. Portanto, $A=\bigcap_{n \geq 0} \bigcup_{k_{j} \geq n} A_{k_{j}}$ e $B=\bigcap_{n \geq 0} \bigcup_{k \geq n} B_{k}$. Seja $x \in A$, então $x \in \bigcup_{k_{j} \geq n} B_{k_{j}}$, para todo $n \geq 0$, concluindo que $x \in B$.

Teorema 5.8 Seja $f: \mathbb{R}^{n} \longrightarrow \mathbb{R}^{n}$ uma aplicação contínua e $\hat{f}$ a extensão de Zadeh restrita ao espaço $\mathcal{F}^{*}\left(\mathbb{R}^{n}\right)$. Se, para todo $B \subset \mathcal{F}^{*}\left(\mathbb{R}^{n}\right)$ não vazio, fechado e limitado, tal que $\hat{f}(B) \subset B$ tivermos $J \neq \emptyset$, onde $J=\omega(B)$, então $\hat{f}$ é assintoticamente suave.

\section{Dem.:}

Basta mostrar que $J$ é compacto, pois como $J=\omega(B)$ e $\hat{f}(B) \subset B$, entäo $J$ atrai $B$.

Como $\mathcal{F}^{*}\left(\mathbb{R}^{n}\right)$ é uma espaço métrico, vamos provar que $J$ é seqüencialmente compacto.

Seja $\left\{u_{j}\right\}$ uma seqüência em $J$. Para cada $\alpha \in[0,1],\left[u_{j}\right]^{\alpha} \subset J_{\alpha}$. Seja $\wp\left(J_{\alpha}\right)$ o conjunto das partes de $J_{\alpha} \cdot \wp\left(J_{\alpha}\right) \subset \mathcal{Q}\left(\mathbb{R}^{n}\right)$ onde $\mathcal{Q}\left(\mathbb{R}^{n}\right)$ é o espaço dos subconjuntos não-vazios e compactos do $\mathbb{R}^{n}$. $\wp\left(J_{\alpha}\right)$ com a métrica de Hausdorff é um espaço métrico completo, conforme foi visto na seção $2.5 \mathrm{e}$ também em [11], página 10. Então, para cada $\alpha \in[0,1]$, existe pelo menos um ponto de acumulação $A_{\alpha} \in \wp\left(J_{\alpha}\right)$ tal que existe uma subseqüencia $\left[u_{j}\right]^{\alpha} \rightarrow A_{\alpha}$ na métrica de Hausdorff, isto é, $h\left(\left[u_{j_{k_{\alpha}}}\right]^{\alpha}, A_{\alpha}\right) \rightarrow 0$ quando $j_{k_{\alpha}} \rightarrow \infty$. 
Vamos escolher $C_{1}=\left\{x_{0}\right\}$, onde $x_{0}$ é um ponto de acumulação de $\left[u_{j_{k_{\alpha}}}\right]^{1}$. Seja $\left\{\left[u_{j_{k}}\right]^{1}\right\}$ uma sequiência que converge para $\left\{x_{0}\right\}$ e para $0 \leq \alpha<1$, seja $\mathcal{A}_{\alpha}=\left\{X \subset \mathbb{R}^{n}\right.$ compacto; $X$ é ponto de acumulação de $\left.\left[u_{j_{k}}\right]^{\alpha} ;\left\{x_{0}\right\} \subset X\right\}$.

Pelo Teorema 5.7, considerando $A_{n}=\left[u_{j_{k_{\alpha}}}\right]^{1}$ e $B_{n}=\left[u_{j_{k}}\right]^{\alpha}$ temos que $\left[u_{j_{k}}\right]^{\alpha} \rightarrow$ $B_{\alpha}$ e $\left\{x_{o}\right\} \subset B_{\alpha}$, para cada $0 \leq \alpha<1, \log 0, \mathcal{A}_{\alpha} \neq \emptyset$.

Para cada $0 \leq \alpha<1$, vamos definir $C_{\alpha}=\bigcap_{X \in \mathcal{A}_{\alpha}} X$

1. $\left\{x_{0}\right\} \subset C_{\alpha}$, para todo $\alpha \in[0,1]$;

2. $C_{\alpha}$ é compacto para todo $\alpha \in[0,1]$;

3. Para todo $\beta<\alpha, C_{\alpha} \subset C_{\beta}$, pelo teorema 5.7.

Então existe $u \in \mathcal{F}^{*}\left(\mathbb{R}^{n}\right)$ tal que $[u]^{\alpha}=C_{\alpha}$ para cada $\alpha \in[0,1]$.

De fato, como vimos,

1. $[u]^{0}=\left\{x_{0}\right\}$ é compacto ;

2. $[u]^{1}=C_{1}$ é não vazio e tem apenas um elemento;

3. Seja a seqüência $\alpha_{k} \nearrow \alpha, \alpha>0$, então $C_{\alpha}=\bigcap C_{\alpha_{k}}$. De fato, - $C_{\alpha} \subset \cap C_{\alpha_{k}}$ : Como $\alpha_{k} \leq \alpha$, para todo $k \geq 0$, então $C_{\alpha} \subset C_{\alpha_{k}}$, para todo $k \geq 0$, isto é, $C_{\alpha} \subset \cap C_{\alpha_{k}}$.

- $C_{\alpha} \supset \bigcap C_{\alpha_{k}}$ : Observe que:

1. $\bigcap_{k \geq 0}\left[u_{n}\right]^{\alpha_{k}}=\left[u_{n}\right]^{\alpha}$, para $n$ fixo, já que $u_{n} \in \mathcal{F}\left(\mathbb{R}^{n}\right)$ e pela Proposição 2.1

2. $\left[u_{n}\right]^{\alpha_{k}} \rightarrow B \in \mathcal{A}_{\alpha_{k}}$, na métrica de Hausdorff.

Então,

$\bigcap C_{\alpha_{k}}=\bigcap_{k \geq 0} \bigcap_{\mathcal{A}_{\alpha_{k}}} \bigcap_{j \geq 0} \bigcup_{l \geq j}\left[u_{n_{l}}\right]^{\alpha_{k}}$

$\subset \bigcap_{k \geq 0} \bigcap_{j \geq 0} U_{l \geq j}\left[u_{n_{l}}\right]^{]_{k}}$

$=\bigcap_{j \geq 0} \bigcap_{k \geq 0} \bigcup_{l \geq j}^{-j}\left[u_{n_{l}}\right]^{\alpha_{k}}$

$=\bigcap_{j \geq 0} \bigcup_{l \geq j} \bigcap_{k \geq 0}\left[u_{n_{l}}\right]^{\alpha_{k}}$

$=\bigcap_{j \geq 0} \bigcup_{l \geq j}\left[u_{n_{l}}\right]^{\alpha} \subset C_{\alpha}$

Portanto, $C_{\alpha}=\bigcap C_{\alpha_{k}}$.

Observe que como $\bigcup_{j=1}^{\infty}\left[u_{j}\right]^{0} \subset J_{0}$ é limitado. Aplicando a Proposição $2.3, u_{j} \rightarrow u$ na métrica $D, \operatorname{logo} J$ é compacto.

Concluindo que $\hat{f}$ é assintoticamente suave. 


\subsection{Outros Exemplos}

Exemplo 5.2 Seja $f: \mathbb{R}^{n} \rightarrow \mathbb{R}^{n}$ contínua tal que $f$ tem um único atrator global $\{0\}$. Então $\hat{f}$ é assintoticamente suave.

De fato, seja $\hat{f}: \mathcal{F}\left(\mathbb{R}^{n}\right) \rightarrow \mathcal{F}\left(\mathbb{R}^{n}\right)$ a extensão de Zadeh de $f$, portanto, conforme o Teorema 3.7 , contínua. Seja $B \subset \mathcal{F}\left(\mathbb{R}^{n}\right), B \neq \emptyset$, fechado, limitado, tal que $\hat{f}(B) \subset B$.

Afirmação 1:

$\{\widehat{0}\}$ atrai $B$.

De fato, para cada $\alpha \in[0,1]$, seja $B_{\alpha}=\overline{\mathrm{U}_{u \in B}[u]^{\alpha}}, B_{\alpha} \subset \mathbb{R}^{n}$ e $B_{\alpha}$ é fechado e limitado, e, portanto, $\{0\}$ atrai $B_{\alpha}$, ou seja, dado $\varepsilon>0$, existe $n_{\alpha} \in \mathbb{N}$ tal que $f^{n}\left(B_{\alpha}\right) \subset N(\{0\}, \varepsilon)$, para todo $n \geq n_{\alpha}$. Mas $B_{\alpha} \subset B_{o}$, para todo $\alpha \in[0,1]$; então, $f^{n}\left(B_{\alpha}\right) \subset f^{n}\left(B_{o}\right)$, para todo $n \in \mathbb{N}$.

Como $f^{n}\left(B_{o}\right) \subset N(\{0\}, \varepsilon)$, para todo $n \geq n_{o}$, para cada $u \in B$, $f^{n}\left([u]^{\alpha}\right) \subset B(\{0\}, \varepsilon)$, para todo $n \geq n_{o}$, ou seja, $\left[\hat{f}^{n}(u)\right]^{\alpha} \subset B(\{0\}, \varepsilon)$, para cada $n \geq n_{o}$, em particular, $\left[\hat{f}^{n}(u)\right]^{\circ} \subset B(\{0\}, \varepsilon)$, para cada $n \geq n_{o}$.

Por outro lado, $h\left(\left[\hat{f}^{n}(u)\right]^{\alpha},\{0\}\right)<\varepsilon$, para todo $n \geq n_{o}$, ou seja,

$$
\{0\} \subset B\left(\left[\hat{f}^{n}(u)\right]^{1}, \varepsilon\right) \text {. }
$$

Pelo Lema 5.2, para cada $u \in B, D\left(\hat{f}^{n}(u),\{\widehat{0}\}\right)<\varepsilon$, para cada $n \geq n_{o}$, ou seja, $D\left(\hat{f}^{n}(B),\{\widehat{0}\}\right)<\varepsilon$, para cada $n \geq n_{o}$, logo $\{\widehat{0}\}$ atrai $B$.

Afirmação 2:

$\{\widehat{0}\} \subset B$, para todo $B \subset \mathcal{F}\left(\mathbb{R}^{n}\right)$, tal que $B \neq \emptyset$, fechado, limitado e $\hat{f}(B) \subset B$.

De fato, como $D\left(\hat{f}^{n}(B),\{\widehat{0}\}\right)<\varepsilon$, para cada $n \geq n_{o}$, temos $\{\widehat{0}\} \subset N\left(\hat{f}^{n}(B), \varepsilon\right)$, para todo $n \geq n_{o}$. Como $\hat{f}(B) \subset B$, segue que

$$
\{\widehat{0}\} \subset N\left(\hat{f}^{n}(B), \varepsilon\right) \subset N(B, \varepsilon), \text { para todo } \varepsilon>0 .
$$

Como $B$ é fechado, segue que $\{\widehat{0}\} \subset B$.

Logo $J=\{\widehat{0}\}$ é o compacto que atrai $B$, o que conclui o exemplo.

Exemplo 5.3 Seja $f: \mathbb{R}^{n} \rightarrow \mathbb{R}^{n}$ contínua com um único atrator compacto global $K$, sendo $K$ não unitário. Neste caso $\hat{f}$ não é assintoticamente suave.

De fato, basta tomar $B=\left\{u \in \mathcal{F}\left(\mathbb{R}^{n}\right) ;[u]^{o} \subset K\right\}$, com diamK $>0$. $B \neq \emptyset$, é fechado, limitado e $\hat{f}(B) \subset B$ e sabemos que $B$ não é compacto. Se $\hat{f}$ fosse assintoticamente suave, existiria $J \subset B, J$ compacto que atrai $B$. Mas $J=\omega(B)=B$, pois $\hat{f}(B) \subset B$, o que é uma contradição, pois sabemos que $B$ só é compacto se $K$ tiver diâmetro zero. 
Exemplo 5.4 Seja $f: \mathbb{R} \rightarrow \mathbb{R}$ e a $\in \mathbb{R}_{+}^{*}$. Para cada $x \in \mathbb{R}, f(x)=$ ax.

A função $f$ é contínua, portanto, como está definida em $\mathbb{R}$, é assintoticamente suave e a extensão de Zadeh desta função, como já vimos no Exemplo 3.3 , é dada por $\hat{f}(u)=a u$, para todo $u \in \mathcal{F}(\mathbb{R})$.

Esta função está associada ao Sistema Discreto:

$$
\left\{\begin{array}{l}
u_{n+1}=a u_{n} \\
u(0)=u_{0}
\end{array}\right.
$$

cuja solução é $u_{n}=a^{n} u_{0}$. Pela definição de multiplicação de um número real por um conjunto fuzzy, se $a \neq 1$, o único ponto fixo é $\hat{0}$; e se $a=1$, qualquer ponto $u \in \mathcal{F}(\mathbb{R})$ é fixo.

A dinâmica deste sistema é a mesma do sistema determinístico, ou seja, $\{\hat{0}\}$ é assintóticamente estável se $a<1$, estável se $a=1$ e instável se $a>1$.

Dado $B \subset \mathcal{F}(\mathbb{R})$ não vazio, fechado e limitado tal que $\hat{f}(B) \subset B$, precisamos achar um $J \subset B$ compacto tal que $J$ atrai $B$.

No caso de $a<1,\{0\}$ é o único atrator global do sistema determinístico, portanto, $\hat{f}$ é assintoticamente suave.

No caso de $a=1$, cada ponto é ponto fixo e para todo $B \subset \mathcal{F}(\mathbb{R})$ fechado e limitado, vamos ter $\hat{f}(B)=B$. Neste caso, só vai existir $J$ compacto quando o próprio $B$ for compacto. Como não vale para todo $B, \hat{f}$ não é assintoticamente suave. Se $a>1$, não existe $B \neq \emptyset$ fechado e limitado tal que $\hat{f}(B) \subset B$, neste caso $\hat{f}$ é assintoticamente suave.

Exemplo 5.5 Seja $f: \mathbb{R}^{2} \rightarrow \mathbb{R}^{2}$, dada por $f(x)=A x$, onde $A=\left[\begin{array}{cc}\lambda_{1} & 0 \\ 0 & \lambda_{2}\end{array}\right]$ e $\lambda_{1}, \lambda_{2} \in \mathbb{R}_{+}^{*}$.

$f$ também é assintoticamente suave e a extensão de Zadeh é dada por $\hat{f}(u)=$ $A u$. Como nos estudos clássicos de sistemas dinâmicos, observa-se que $\hat{f}$ é assintoticamente suave se, e somente se, $\left|\lambda_{i}\right| \neq 1$, para todo $i \in\{1,2\}$.

\subsection{Dissipatividade em $\mathcal{F}\left(\mathbb{R}^{n}\right)$}

Baseado no teorema 4.3 e lembrando que toda função contínua $f: \mathbb{R}^{n} \rightarrow \mathbb{R}^{n}$ é assintoticamente suave, temos o seguinte resultado: 
Teorema 5.9 Seja $f: \mathbb{R}^{n} \rightarrow \mathbb{R}^{n}$ continua, dissipativa por pontos, cujas órbitas de conjuntos limitados são limitadas, então existe um atrator global conexo $K$.

Vamos considerar o seguinte conjunto:

$$
J_{K}=\left\{u \in \mathcal{F}\left(\mathbb{R}^{n}\right) ;[u]^{o} \subset K\right\} \text {. }
$$

Já vimos na seção 5.2 que $J_{K}$ é fechado e limitado. Vamos mostrar que ele atrai pontos em $\mathcal{F}\left(\mathbb{R}^{n}\right)$, ou seja, que a extensão de Zadeh $\hat{f}$ é dissipativa por pontos. Mas antes, vamos demonstrar a seguinte proposição:

Proposição 5.2 Sejam $f: \mathbb{R}^{n} \rightarrow \mathbb{R}^{n}$ contínua com um atrator global conexo, $C \subset \mathbb{R}^{n}$ um conjunto compacto e $C^{\prime}=\bigcup_{c \in C} \omega(c)$. Então $f^{n}(C) \rightarrow C^{\prime}$ na métrica de Hausdorff.

\section{Dem.:}

Temos que mostrar que dado $\epsilon>0$, existe $n_{o} \in \mathbb{N}$ tal que

$$
h\left(f^{n}(C), C^{\prime}\right)<\epsilon, \text { para todo } n \geq n_{o} .
$$

Seja $c \in C$, então existe $c^{\prime} \in C^{\prime}$ tal que $f^{n}(c) \rightarrow c^{\prime}$, ou seja, dado $c \in C$ e $\epsilon>0$, existe $n_{c} \in \mathbb{N}$ e $c^{\prime} \in C^{\prime}$ tal que $d\left(f^{n}(c), c^{\prime}\right)<\epsilon$, para todo $n \geq n_{c}$, ou ainda, inf $d\left(f^{n}(c), C^{\prime}\right)<\epsilon$, para todo $n \geq n_{c}$.

Por outro lado, seja $c^{\prime} \in C^{\prime}$, então existe $c \in C$ tal que $c^{\prime}=\omega(c)$, ou seja, dado $\epsilon>0$, existe $c \in C$ e $n_{c}^{\prime} \in \mathbb{N}$ tal que $f^{n}(c) \rightarrow c^{\prime}$, ou seja, inf $d\left(f^{n}(C), c^{\prime}\right)<\epsilon$, para todo $n^{\prime} \geq n_{c}$. Como $C$ e $C^{\prime}$ são compactos, existe $n_{o} \in \mathbb{N}$ tal que $h\left(f^{n}(C), C^{\prime}\right)<\epsilon$, para todo $n \geq n_{o}$.

Teorema 5.10 Seja $f: \mathbb{R}^{n} \rightarrow \mathbb{R}^{n}$ contínua, dissipativa por pontos, cujas órbitas de conjuntos limitados são limitadas, então a extensão de Zadeh $\hat{f}: \mathcal{F}\left(\mathbb{R}^{n}\right) \rightarrow \mathcal{F}\left(\mathbb{R}^{n}\right)$ é dissipativa por pontos. 


\section{Dem.:}

Pelo Teorema 5.9, existe um atrator global conexo $K$ para o sistema dinâmico gerado pela função $f$. Seja $u \in \mathcal{F}\left(\mathbb{R}^{n}\right)$, como $K$ é atrator global conexo, pela Proposição 5.2, dado $\epsilon>0$, existe $n_{o} \in \mathbb{N}$ tal que

$$
h\left(f^{n}\left([u]^{o}\right), K\right)<\epsilon, \text { para todo } n \geq n_{o}
$$

ou seja,

$$
\inf _{\alpha \in[0,1]} h\left(f^{n}\left([u]^{\alpha}\right), K\right)<\epsilon \text {, para todo } n \geq n_{o} .
$$

Seja $J_{K}$ como acima, então, $D\left(\hat{f^{n}}(u), J_{K}\right)<\epsilon$, para todo $n \geq n_{o}$, ou seja, $J_{K}$ atrai pontos de $\mathcal{F}\left(\mathbb{R}^{n}\right)$, o que implica que $\hat{f}$ é dissipativa por pontos.

Alguns exemplos:

Exemplo 5.6 Seja $f: \mathbb{R} \rightarrow \mathbb{R}$ uma função contínua com um atrator global $K=[a, b]$ como abaixo:

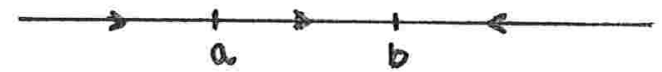

Então $\hat{f}: \mathcal{F}(\mathbb{R}) \rightarrow \mathcal{F}(\mathbb{R})$ é dissipativa por pontos e o conjunto

$$
B_{K}=\left\{u \in \mathcal{F}(\mathbb{R}) ;[u]^{o} \subset[a, b]\right\}
$$

só é compacto se $a=b$, ou seja, $\hat{f}$ só é assintoticamente suave se $a=b$.

Exemplo 5.7 Seja $f: \mathbb{R}^{2} \rightarrow \mathbb{R}^{2}$ uma função dada por $f(x)=A x$, onde $A=\left[\begin{array}{cc}\lambda_{1} & 0 \\ 0 & \lambda_{2}\end{array}\right] \operatorname{com} \lambda_{1}<1$ e $\lambda_{2}<1$.

Neste caso sabemos que $\{0\}$ é atrator global de $f$ e que as órbitas de conjuntos limitados são limitadas. Então $\hat{f}: \mathcal{F}\left(\mathbb{R}^{2}\right) \rightarrow \mathcal{F}\left(\mathbb{R}^{2}\right)$ é dissipativa por pontos. Como já vimos que $\hat{f}$ é assintoticamente suave, pelo teorema 4.3 , segue que $\{\hat{0}\}$ é o atrator global conexo de $\hat{f}$. 
Exemplo 5.8 Seja $f: \mathbb{R}^{2} \rightarrow \mathbb{R}^{2}$ a função dada por

$$
f(x, y)=\left(y+x\left(1-x^{2}-y^{2}\right),-x+y\left(1-x^{2}-y^{2}\right)\right)
$$

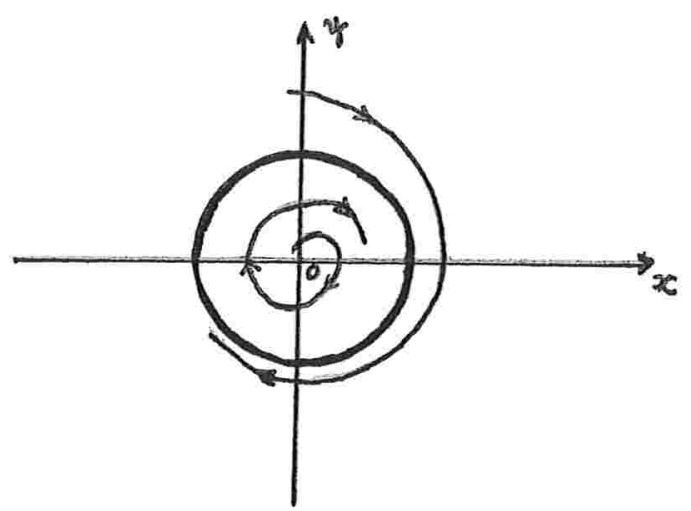

$S^{1}$ é um atrator global conexo e as órbitas de conjuntos limitados são limitadas, portanto $\hat{f}: \mathcal{F}\left(\mathbb{R}^{2}\right) \rightarrow \mathcal{F}\left(\mathbb{R}^{2}\right)$ é dissipativa por pontos e o conjunto atrator é o $B_{K}=\left\{u \in \mathcal{F}\left(\mathbb{R}^{2}\right) ;[u]^{o} \subset S^{1}\right\}$. Mas $\hat{f}$ não é assintoticamente suave pois $S^{1}$ não é um conjunto unitário.

\subsection{Projetos}

Existem vários projetos a serem desenvolvidos, mas aqui vamos falar um pouco da idéia que temos de estudar Inclusões Diferenciais Fuzzy.

Sabemos bem da importância das Equações Diferenciais, tanto do ponto de vista teórico como das aplicações, mas, em muitos casos, estas equações se distanciam das aplicações quando descrevem certos sistemas de evolução. Uma maneira de modelar tais problemas seria através de Inclusões Diferenciais, ou seja, encontrar $x:[0, T] \rightarrow \mathbb{R}^{n}$ tal que

$$
x^{\prime}(t) \in G(t, x(t)),
$$

onde $G$ é uma função multivaluada.

A nossa intenção é estudar a teoria de inclusões diferenciais no contexto fuzzy. Existem várias formulações de Inclusões Diferenciais Fuzzy e Equações Diferenciais Fuzzy. No nosso caso, deveríamos considerar como Equação Diferencial Fuzzy, uma equação do tipo:

$$
u^{\prime}(t)=G(t, u(t))
$$


onde $u^{\prime}(t)$ é a derivada segundo Hukuhara e $G: \mathbb{R}^{n} \rightarrow \mathcal{F}\left(\mathbb{R}^{n}\right)$ uma multifunção fuzzy.

Então o problema de Inclusão Diferencial a estudar seria

$$
u^{\prime}(\dot{t}) \in G(t, u(t))
$$

Este problema pode ser interpretado de algumas maneira diferentes. Uma interpretação seria

$$
\text { ' } \chi_{\left\{u^{\prime}(t)\right\}} \subset G(t, u(t)),
$$

o que seria um tanto trivial, pois neste caso o estudo se resumiria ao estudo da seguinte inclusão diferencial:

$$
u^{\prime}(t) \in[G(t, u(t))]^{1} .
$$

Outra interpretação, sugerida por Yuanguo Zhu [39], seria considerar uma função $\alpha: \mathbb{R}^{n} \rightarrow[0,1]$ e o problema seria definido como:

$$
u^{\prime}(t) \in[G(t, u(t))]^{\alpha(u(t))} .
$$

Estudar propriedades das Inclusões Diferenciais Fuzzy tais como: estabilidade, periodicidade, etc e comparar resultados dentre as várias interpretações são alguns dos pontos relevantes deste projeto de estudos. 


\section{Bibliografia}

[1] Bassanezi, R.C.; Barros, L.C.; Tonelli, P.A. - Attractors and Asymptotic Stability for Fuzzy Dynamical Systems - Fuzzy Sets and Systems 113, $473-483,2000$.

[2] Barros, L.C. - Modelos Determinísticos com Parâmetros Subjetivos - Dissertação de Mestrado - Instituto de Matemática e Estatística da UNICAMP, 1992.

[3] Barros, L.C. - Sobre Sistemas Dinâmicos Fuzzy - Teoria e Aplicações -Tese de Doutorado - Instituto de Matemática e Estatística da UNICAMP, 1997.

[4] Barros, L. C. ; Bassanezi, R. C. ; Tonelli, P. A. - On the continuity of Zadeh's Extension - Proceedings Seventh IFSA World Congress, Vol. II, 3-8, Prague, 1997.

[5] Barros, L. C. ; Souza, S. A. O. ; Tonelli, P. A. - Asymptotic Smoothness of the Zadeh's Extensions - Preprint submetido ao J. of Math. Analysis. and Appl., 1999.

[6] Barros, L. C. ; Souza, S. A. O. ; Tonelli, P. A. - Two Cases of Asymptotic Smoothness for Fuzzy Dynamical Systems - Relatório Técnico RTMAP-0001 - Instituto de Matemática e Estatística da USP - São Paulo, Fevereiro, 2000.

[7] Black, Max - Vagueness: An Exercise in Logical Analysis - Phylosophy of Science 4, 427-455, 1937.

[8] Brumley, W. E. - On the Asymptotic Behavior of Solutions of Differential Difference Equations of Neutral Type - J. of Differential Equations 7, 175-188, 1970. 
[9] Cabrelli, C. A.; Forte, B.; Molter, U.M. e Vrscay,E.R. - Iterated Fuzzy Set Systems: A New Approach to the Inverse Problem for Fractals and Others Sets - Journal of Math. Analysis and Applications 171, 79-100, 1992.

[10] Cooperman, G. - $\alpha$-Condensing Maps and Dissipative Processes- Ph. D. Thesis, Brown University, Providence, R. I., 1978.

[11] Diamond, P. e Kloeden, P. - Metric Spaces of Fuzzy Sets: Theory and Applications - World Scientific Pub, 1994.

[12] Diamond, P. - Time-Dependent Differential Inclusions, Cocycles Attractors and Fuzzy Differential Equations - IEEE Transactions on Fuzzy Systems 7, 734-740, 1999.

[13] Gregori, V. e Romaguera, S. - Some Properties of Fuzzy Metric Spaces - Fuzzy Sets and Systems 115, $485-489,2000$.

[14] Hale, J. K. - Asymptotic Behavior of Dissipative Systems- Math. Surveys and Monographs 25, American Mathematical Society, Providence, 1988.

[15] Hausdorff, F. - Set Theory - New York, Chelsea, 1957.

[16] Kaleva, O. - The Cauchy Problem for Fuzzy Differential Equations Fuzzy Sets and Systems 35, 389-396, 1986.

[17] Kaleva, O. - On the Convergence of Fuzzy Sets - Fuzzy Sets and Systems 17, 53-65, 1985.

[18] Klir, G.J. e Bo Yuan - Fuzzy Sets and Fuzzy Logics - New Jersey, Prentice-Hall, 1995.

[19] Kloeden, P.E. - Compact Supported Endographs and Fuzzy Sets - Fuzzy Sets and Systems 4, 193-201, 1960.

[20] Kosko, Bart - Fuzzy Thinking - New York, Hyperion, 1993.

[21] Lima, Elon L. - Espaços Métricos - Rio de Janeiro, Instituto de Matemática Pura e Aplicada, CNPq, 1977. 
[22] Negoita, C.V. e Ralescu, D.A. - Applications of Fuzzy Sets to System Analysis - New York, Wiley, 1975.

[23] Nguyen,H.T. e Walker, E.A. - A First Course in Fuzzy Logic - New Mexico, CRC Press, 1997.

[24] Ortega, N.R.S., Barros, L.C. e Massad,E. - An Application of the Extension Principle of Fuzzy Epidemic Models - preprint submetido ao Fuzzy Sets and Systems, 2000.

[25] Pedrycz,W. e Gomide F. - An Introduction to Fuzzy Sets: Analysis and Design - A Bradford Book, 1998.

[26] Puri, M. L. e Ralescu, D. A. - Fuzzy Random Variables - J. of Mathematical Analysis and Applications 114, 409-422, 1986.

[27] Quelho, E. - Sobre A-Convergências - Tese de Doutorado - Instituto de Matemática e Estatística da UNICAMP, 1989.

[28] Reznik, L. - Fuzzy Controlers - England, Newnes, 1997.

[29] Rojas-Medar, M. e Roman-Flores, H. - On the Equivalence of Convergences of Fuzzy Sets - Fuzzy Sets and Systems 80, 217-224, 1996.

[30] Roman-Flores, H.; Barros, L. e Bassanezi, R. - A Note on Zadeh's Extensions - Fuzzy Sets and Systems 117, 327 - 331, 2001.

[31] Roman-Flores, H.; Flores-Franulic, A.; Rojas-Medar, M. e Bassanezi, R. C. - On the Compactness of $E(X)$ - Technical Report, RP 78/97, Instituto de Matemática e Estatística da UNICAMP, Outubro, 1997.

[32] Roman-Flores, H. e Rojas-Medar, M. - Embedding of Level-continuous Fuzzy Sets on Banach Spaces - Technical Report, RP 46/99, Instituto de Matemática e Estatística da UNICAMP, Julho, 1999.

[33] Roos, Timothy - Fuzzy Logic with Engennering Application - Hardcover, 1994.

[34] Rudin,W. - Real and Complex Analysis -New York, Addison Wesley, 1976. 
[35] Sanchez, E. - Medical Diagnosis and Composite Fuzzy Relations. In: Gupta, M.M., R.K. Ragade e R.R. Yager eds., Advances in Fuzzy Set Theory and Applications, 437-444, New York, North Holland, 1979.

[36] Seikkala, S. - On the Fuzzy Initial Value Problem - Fuzzy Sets and Systems 24, 319-330, 1987.

[37] Yager, R.R. - A Characterization of the Extension Principle - Fuzzy Sets and Systems 18, 205-217, 1986.

[38] Zadeh, L. A. - Fuzzy Sets - Information and Control 8, 338-353, 1965.

[39] Zhu, Yuanguo e Rao, Ling - Differential Inclusions for Fuzzy Maps Fuzzy Sets and Systems 112, 257-261, 2000. 